Geometry $\&$ Topology

Volume 6 (2002) 609-647

Published: 6 December 2002

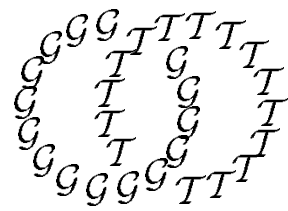

\title{
Boundary curves of surfaces with the 4-plane property
}

\author{
TAO LI \\ Department of Mathematics, Oklahoma State University \\ Stillwater, OK 74078, USA \\ Email: tli@math.okstate.edu \\ URL: http://www.math.okstate.edu/ ${ }^{\sim}$ tli
}

\begin{abstract}
Let $M$ be an orientable and irreducible 3-manifold whose boundary is an incompressible torus. Suppose that $M$ does not contain any closed nonperipheral embedded incompressible surfaces. We will show in this paper that the immersed surfaces in $M$ with the 4-plane property can realize only finitely many boundary slopes. Moreover, we will show that only finitely many Dehn fillings of $M$ can yield 3-manifolds with nonpositive cubings. This gives the first examples of hyperbolic 3-manifolds that cannot admit any nonpositive cubings.
\end{abstract}

AMS Classification numbers Primary: 57M50

Secondary: 57M25, 57N10, 57M07

Keywords: 3-manifold, immersed surface, nonpositive cubing, 4-plane property, immersed branched surface.

Proposed: Cameron Gordon

Seconded: Walter Neumann, Michael Freedman
Received: 23 March 2001

Revised: 15 March 2002

(c) Geometry $\mathcal{G}$ Topology $\mathcal{P}$ ublications 


\section{Introduction}

A closed irreducible 3-manifold is called Haken if it contains a two-sided incompressible surface. Waldhausen has proved topological rigidity for Haken 3-manifolds [30], ie, if two Haken 3-manifolds are homotopically equivalent, then they are homeomorphic. However, a theorem of Hatcher [15] implies that, in a certain sense, most $3-$ manifolds are not Haken. Immersed $\pi_{1}$-injective surfaces are a natural generalization of incompressible surfaces, and conjecturally, 3 -manifolds that contain $\pi_{1}$-injective surfaces have the same topological and geometric properties as Haken 3-manifolds. Another related major conjecture in 3-manifold topology is that any 3-manifold with infinite fundamental group contains a $\pi_{1}$-injective surface.

Hass and Scott [14] have generalized Waldhausen's theorem by proving topological rigidity for 3 -manifolds that contain $\pi_{1}$-injective surfaces with the 4 -plane and 1-line properties. A surface in a 3 -manifold is said to have the $n$-plane property if its preimage in the universal cover of the 3-manifold is a union of planes, and among any collection of $n$ planes, there is a disjoint pair. The $n$-plane property is a good way to measure the combinatorial complexity of an immersed surface. It has been shown [28] that any immersed $\pi_{1}$-injective surface in a hyperbolic $3-$ manifold satisfies the $n$-plane property for some $n$.

In this paper, we use immersed branched surfaces to study surfaces with the 4-plane property. Branched surfaces have been used effectively in the studies of incompressible surfaces and laminations [9, 11]. Many results in 3-manifold topology (eg Hatcher's theorem [15]) are based on the theory of branched surfaces. We define an immersed branched surface in a 3 -manifold $M$ to be a local embedding to $M$ from a branched surface that can be embedded in some 3-manifold (see definition 2.4). Immersed branched surfaces are also used in [21]. Using lamination techniques and immersed branched surfaces, we show:

Theorem 1 Let $M$ be a closed, irreducible and non-Haken 3-manifold. Then there is a finite collection of immersed branched surfaces such that any surface in $M$ with the 4-plane property is fully carried by an immersed branched surface in this collection.

This theorem generalizes a fundamental result of Floyd and Oertel [9] in the theory of embedded branched surfaces. One important application of the theorem of Floyd and Oertel is the proof of a theorem of Hatcher [15], which says that incompressible surfaces in an orientable and irreducible 3 -manifold with torus boundary can realize only finitely many slopes. A slope is the isotopy 
class of a nontrivial simple closed curve in a torus. We say that a surface in a 3 -manifold with torus boundary can realize a slope $s$ if the boundary of this surface consists of simple closed curves with slope $s$ in the boundary torus of the 3-manifold. If an immersed surface can realize a slope $s$, then it extends to a closed surface in the closed manifold obtained by Dehn filling along the slope $s$. However, Hatcher's theorem is not true for immersed $\pi_{1}$-injective surfaces in general, since there are many 3 -manifolds $[2,26,3,22]$ in which $\pi_{1}$-injective surfaces can realize infinitely many slopes, and in some cases, can realize every slope. Using Theorem 1, we will show that surfaces with the 4-plane property are, in a sense, like incompressible surfaces. Note that many 3 -manifolds satisfy the hypotheses in Theorems 2 and 3, such as hyperbolic punctured-torus bundles $[7,8]$ and hyperbolic 2-bridge knot complements [16].

Theorem 2 Let $M$ be an orientable and irreducible 3-manifold whose boundary is an incompressible torus, and let $\mathcal{H}$ be the set of injective surfaces that are embedded along their boundaries and satisfy the 4-plane property. Suppose that $M$ does not contain any nonperipheral closed (embedded) incompressible surfaces. Then the surfaces in $\mathcal{H}$ can realize only finitely many slopes.

Aitchison and Rubinstein have shown that if a 3-manifold has a nonpositive cubing, then it contains a surface with the 4-plane and 1-line properties [1], and hence topological rigidity holds for such 3-manifolds. Nonpositive cubings, which were first introduced by Gromov [12], are an important example of CAT(0) structure. A 3-manifold is said to admit a nonpositive cubing if it is obtained by gluing cubes together along their square faces under the following conditions: (1) For each edge, there are at least four cubes sharing this edge; (2) for each vertex, in its link sphere, any simple 1-cycle consisting of no more than three edges must consist of exactly three edges, and must bound a triangle. Mosher [23] has shown that if a 3-manifold has a nonpositive cubing, then it satisfies the weak hyperbolization conjecture, ie, either it is negatively curved in the sense of Gromov or its fundamental group has a $\mathbb{Z} \oplus \mathbb{Z}$ subgroup.

Nonpositively cubed 3-manifolds have very nice topological and geometric properties. A natural question, then, is how large the class of such 3-manifolds is. Aitchison and Rubinstein have constructed many examples of such 3-manifolds, and only trivial examples, such as manifolds with finite fundamental groups, were known not to admit such cubings. At one time, some people believed that every hyperbolic 3-manifold admits a nonpositive cubing. In this paper, we give the first nontrivial examples of 3-manifolds, in particular, the first examples of hyperbolic 3-manifolds that cannot admit any nonpositive cubings. In 
fact, Theorem 3 says that, in a certain sense, most 3-manifolds do not have such a cubing.

Theorem 3 Let $M$ be an orientable and irreducible 3-manifold whose boundary is an incompressible torus. Suppose that $M$ does not contain any closed nonperipheral (embedded) incompressible surfaces. Then only finitely many Dehn fillings on $M$ can yield 3-manifolds that admit nonpositive cubings.

Acknowledgments This paper is a part of my thesis. I would like to thank my advisor Dave Gabai, who introduced this subject to me, for many very helpful conversations. I am also very grateful to Yanglim Choi for a series of meetings about his thesis and for his work on immersed branched surfaces.

\section{Hatcher's trick}

A branched surface in a 3-manifold is a closed subset locally diffeomorphic to the model in Figure 2.1 (a). A branched surface is said to carry a surface (or lamination) $S$ if, after homotopies, $S$ lies in a fibered regular neighborhood of $B$ (as shown in Figure $2.1(\mathrm{~b})$ ), which we denote by $N(B)$, and is transverse to the interval fibers of $N(B)$. We say that $S$ is fully carried by a branched surface $B$ if it meets every interval fiber of $N(B)$. A branched surface $B$ is said to be incompressible if it satisfies the following conditions: (1) The horizontal boundary of $N(B)$, which we denote by $\partial_{h} N(B)$, is incompressible in the complement of $N(B)$, and $\partial_{h} N(B)$ has no sphere component; (2) $B$ does not contain a disk of contact; (3) there is no monogon (see [9] for details).

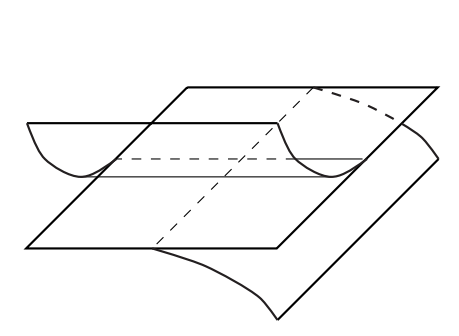

(a)

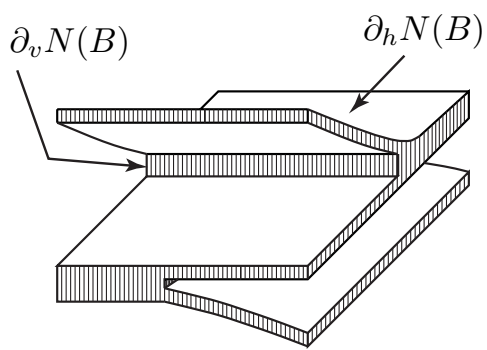

(b)

Figure 2.1

Theorem 2.1 (Floyd-Oertel) Let $M$ be a compact irreducible 3-manifold with incompressible boundary. Then there are finitely many incompressible 
branched surfaces such that every incompressible and $\partial$-incompressible surface is fully carried by one of these branched surfaces. Moreover, any surface fully carried by an incompressible branched surface is incompressible and $\partial-$ incompressible.

Using this theorem and a simple trick, Hatcher has shown [15] that given a compact, irreducible and orientable 3-manifold $M$ whose boundary is an incompressible torus, incompressible and $\partial$-incompressible surfaces in $M$ can realize only finitely many boundary slopes. An immediate consequence of Hatcher's theorem is that if $M$ contains no closed nonperipheral incompressible surfaces, then all but finitely many Dehn fillings on $M$ yield irreducible and non-Haken 3 -manifolds. To prove Hatcher's theorem, we need the following lemma [15].

Lemma 2.2 (Hatcher) Let $T$ be a torus and $\tau$ be a train track in $T$ that fully carries a union of disjoint and nontrivial simple closed curves. Suppose that $\tau$ does not bound a monogon. Then $\tau$ is transversely orientable.

In Theorem 2.1, if $\partial M$ is a torus, the boundaries of those incompressible branched surfaces are train tracks that satisfy the hypotheses in Lemma 2.2. This lemma together with a trick of Hatcher prove the following.

Theorem 2.3 (Hatcher) Let $M$ be a compact, orientable and irreducible 3manifold whose boundary is an incompressible torus. Suppose that $(B, \partial B) \subset$ $(M, \partial M)$ is an incompressible branched surface. If $S_{1}$ and $S_{2}$ are two embedded surfaces fully carried by $B$, then $\partial S_{1}$ and $\partial S_{2}$ have the same slope in the torus $\partial M$. Moreover, the incompressible and $\partial$-incompressible surfaces in $M$ can realize only finitely many slopes.

Proof Since $M$ is orientable, the normal direction of $\partial M$ and the transverse orientation of $\partial B$ uniquely determine an orientation for every curve carried by $\partial B$. Since $S_{i}$ is fully carried by $B$, every component of $\partial S_{i}(i=1$ or 2$)$ with this induced orientation represents the same element in $H_{1}(\partial M)$. If $\partial S_{1}$ and $\partial S_{2}$ have different slopes, they must have a nonzero intersection number. There are two possible configurations for the induced orientations of $\partial S_{1}$ and $\partial S_{2}$ at endpoints of an arc $\alpha$ of $S_{1} \cap S_{2}$, as shown in Figure 2.2. In either case, the two ends of $\alpha$ give points of $\partial S_{1} \cap \partial S_{2}$ with opposite intersection numbers. Thus, the intersection number $\partial S_{1} \cdot \partial S_{2}=0$. So, they must have the same slope. The last assertion of the theorem follows from the theorem of Floyd and Oertel. 

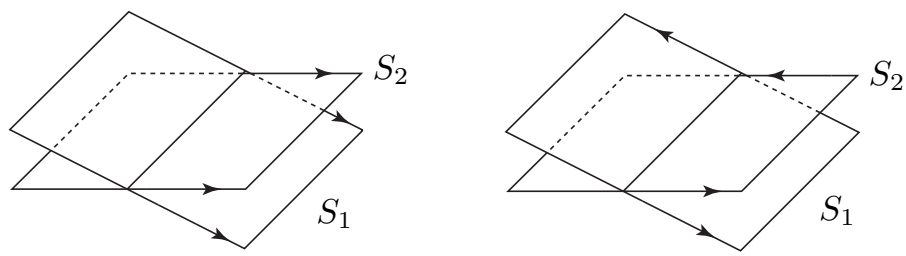

Figure 2.2

In order to apply the trick of intersection numbers in the proof of Hatcher's theorem, we do not need the surfaces $S_{1}$ and $S_{2}$ to be embedded. In fact, if $S_{1}$ and $S_{2}$ are immersed $\pi_{1}$-injective surfaces that are embedded along their boundaries and transversely intersect the interval fibers of $N(B)$, then $\partial S_{1}$ and $\partial S_{2}$ must have the same slope by the same argument. This is the starting point of this paper. In fact, even the branched surface $B$ can be immersed. An obstruction to applying Hatcher's trick is the existence of a local picture as in Figure 2.3 in $B$. Next, we will give our definition of immersed branched surfaces so that we can apply Hatcher's trick to immersed surfaces.

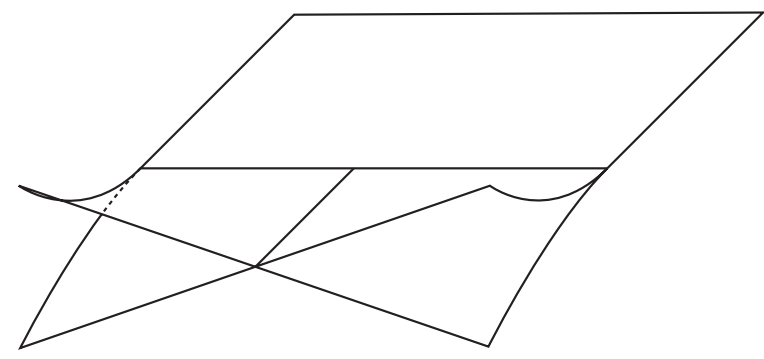

Figure 2.3

Definition 2.4 Let $B$ be a branched surface properly embedded in some compact 3-manifold, ie, the local picture of $B$ in this manifold is as in Figure 2.1 (a). Let $i: B \rightarrow M$ (respectively $i: N(B) \rightarrow M$ ) be a map from $B$ (respectively $N(B)$ ) to a 3-manifold $M$. We call $i(B)$ an immersed branched surface in $M$ if the map $i$ is a local embedding. An immersed surface $j: S \rightarrow M$ (or simply $S$ ) is said to be carried by $i(B)$ (or $B$ ) if, after some homotopy in $M, j=i \circ h$, where $h: S \rightarrow N(B)$ is an embedding and $h(S)$ is transverse to the interval fibers of $N(B)$. We say that it is fully carried by $i(B)$ if $h(S)$ transversely intersects every $I$-fiber of $N(B)$.

If $i: B \rightarrow M$ is an immersed branched surface, then $i(B)$ contains no local picture as in Figure 2.3 by definition. The following proposition is an extension 
of Hatcher's theorem, and its proof is simply an application of Hatcher's trick to immersed branched surfaces.

Proposition 2.5 Let $M$ be a compact, orientable and irreducible 3-manifold whose boundary is an incompressible torus. Let $S_{1}$ and $S_{2}$ be immersed $\pi_{1-}$ injective surfaces fully carried by an immersed branched surface $i: B \rightarrow M$. Suppose that $\left.i\right|_{\partial B}$ is an embedding and $i(\partial B)$ does not bound a monogon. Then $\partial S_{1}$ and $\partial S_{2}$ have the same slope.

\section{Cross disks}

We have seen in section 2 that Hatcher's trick can be applied to immersed branched surfaces. However, we also need finiteness of the number of branched surfaces, as in the theorem of Floyd and Oertel, to get interesting results. This is impossible in general because there are many examples of 3 -manifolds in which immersed $\pi_{1}$-injective surfaces can realize infinitely many slopes. In this section, we will show that one can generalize the theorem of Floyd and Oertel to immersed surfaces with a certain property and such immersed surfaces can realize only finitely many slopes.

Using normal surface theory, it is very easy to get finiteness (of the number of branched surfaces) in the case of embedded incompressible surfaces. For any triangulation of a 3-manifold, an incompressible surface can be put in KneserHaken normal form $[20,13]$. There are 7 types of normal disks in a tetrahedron, 4 triangular types and 3 quadrilateral types. By identifying all the normal disks (in the intersection of the surface with a tetrahedron) of the same type to a branch sector, we can naturally construct a branched surface fully carrying this embedded normal surface, and the finiteness follows from the compactness of the 3-manifold (see [9] for details). However, in the case of immersed surfaces, we cannot do this, although immersed $\pi_{1}$-injective surfaces can also be put in normal form. If we simply use the construction in [9] and identify all the normal disks (in an immersed surface) of the same type to a branch sector, we may get a local picture like that in Figure 2.3, which makes Hatcher's argument fail.

Suppose that $S$ is a $\pi_{1}$-injective surface in a 3 -manifold $M$ with a triangulation $\mathcal{T}$. Using normal surface theory, we can put $S$ in normal form. Let $\widetilde{M}$ be the universal cover of $M, \pi: \widetilde{M} \rightarrow M$ be the covering map, $\widetilde{S}=\pi^{-1}(S)$, and $\widetilde{\mathcal{T}}$ be the induced triangulation of $\widetilde{M}$. For any arc $\alpha$ in $M$ (or $\widetilde{M}$ ) whose interior does not intersect the 1 -skeleton $\mathcal{T}^{(1)}$, we define the length of $\alpha$ to be $\left|\operatorname{int}(\alpha) \cap \mathcal{T}^{(2)}\right|$, where $\operatorname{int}(E)$ denotes the interior of $E$ and $|E|$ denotes 
the number of connected components of $E$. Moreover, we define the distance between points $x$ and $y, d(x, y)$, to be the minimal length of all such arcs connecting $x$ to $y$. In this paper, we will always assume our curves do not intersect the 1-skeleton of the triangulation, and we always use the distance defined above unless specified.

Let $f: F \rightarrow M$ be an immersed surface. We define the weight of $f(F)$ to be $\left|f^{-1}\left(\mathcal{T}^{(1)}\right)\right|$. A normal (immersed) surface $f: F \rightarrow M$ is said to have least weight if $\left|f^{-1}\left(\mathcal{T}^{(1)}\right)\right|$ is minimal in the homotopy class of $f$. Let $f:(F, \partial F) \rightarrow$ $(M, \partial M)\left(F \neq S^{2}\right.$ or $\left.P^{2}\right)$ be a $\pi_{1}$-injective map, and $M_{F}$ be the cover of $M$ such that $\pi_{1}\left(M_{F}\right)$ equals $f_{*}\left(\pi_{1}(F)\right)$. We will suppose that the lift of $f$ into $M_{F}$ is an embedding (note that this is automatic if $f$ is least area in the smooth or PL sense $[14,18])$. Thus, the preimage of $f(F)$ in $\widetilde{M}$ consists of an embedded simply connected surface $\Pi$ which covers $F$ in $M_{F}$ and the translates of $\Pi$ by $\pi_{1}(M)$. We say $f$ has the $n$-plane property if, given any collection of $n$ translates of $\Pi$, there is always a disjoint pair. We say that $\Pi$ above has least weight if every disk in $\Pi$ has least weight among all the disks in $\widetilde{M}$ with the same boundary. It follows from Theorem 5 of [18] or Theorem 3.4 of [10] that $f$ can be chosen so that $\Pi$ has least weight, and hence any translate of $\Pi$ has least weight. By Theorem 8 of [18] (or Theorem 6.3 of [10]), if there is a map $g$ in the homotopy class of $f$ having the $n$-plane property, then we can choose $f$ so that $f$ is a normal surface with least weight, $\Pi$ has least weight, and $f$ also has the $n$-plane property. Note that $F$ may be a surface with boundary and $\Pi$ may not be a plane $\mathbb{R}^{2}$, but since the interior of $\Pi$ is a plane, to simplify notation, we will call each translate of $\Pi$ a plane in the preimage of $f(F)$ (in $\widetilde{M})$ throughout this paper.

A normal homotopy is defined to be a smooth map $H: F \times[0,1] \rightarrow M$ so that for each $t \in[0,1]$, the surface $F_{t}$ given by $\left.H\right|_{F \times\{t\}}$ is a normal surface. Note that the weight of $F_{t}$ is fixed in a normal homotopy.

In this paper, we will assume that our 3-manifolds are compact and irreducible, and our immersed surfaces, when restricted to the boundary, are embedded. We will also assume that our injective surfaces are normal and have least weight, and any plane in their preimages in the universal cover of the 3-manifold also has least weight. To simplify notation, we will not distinguish $f: F \rightarrow M, F$ and $f(F)$ unless necessary, and we will always denote the preimage of $f(F)$ in the universal cover $\widetilde{M}$ by $\widetilde{F}$ throughout this paper.

Definition 3.1 Let $f: F \rightarrow M$ be a $\pi_{1}$-injective and least weight normal surface, and $\Pi$ be a plane in the preimage of $f(F)$ in $\widetilde{M}$ as above. Each plane 
in the preimage of $f(F)$ in $\widetilde{M}$ is a translate of $\Pi$ by an element in $\pi_{1}(M)$. Let $F_{1}$ and $F_{2}$ be two such planes in $\widetilde{M}$. Suppose that $D_{1}$ and $D_{2}$ are two embedded subsurfaces in $F_{1}$ and $F_{2}$ respectively. We say that $D_{1}$ and $D_{2}$ are parallel if there is a normal homotopy $H: D \times I \rightarrow \widetilde{M}$ such that $H(D, 0)=D_{1}$, $H(D, 1)=D_{2},\left.H\right|_{D \times\{t\}}$ is an embedding for each $t \in I$, and $H$ fixes the 2skeleton, ie, if $H(x, y) \in \widetilde{\mathcal{T}}^{(i)}$ then $H(x, I) \subset \widetilde{\mathcal{T}}^{(i)}(i=1,2)$. We call $D_{1} \cup D_{2}$ a cross disk if $D_{1}$ and $D_{2}$ are parallel disks, $F_{1} \neq F_{2}$, and $F_{1} \cap F_{2} \neq \emptyset$. We call $D_{i}(i=1,2)$ a component of the cross disk $D_{1} \cup D_{2}$. Let $H$ be the normal homotopy above. We call $H(p, 0) \cup H(p, 1)$ a pair of points (respectively arcs, disks) in the cross disk, for any point (respectively arc, disk) $p$ in $D$. A cross disk $D_{1} \cup D_{2}$ (or the disk $D_{1}$ ) is said to have size at least $R$ if there exists a point $x \in D_{1}$ such that length $(\alpha) \geq R$ for any normal arc $\alpha \subset D_{1}$ connecting $x$ to $\partial D_{1}-\partial \widetilde{M}$, and we call the normal disk of $T \cap D_{1}$ that contains $x$ a center of the cross disk, where $T$ is a tetrahedron in the triangulation. To simplify notation, we also call $\pi\left(D_{1} \cup D_{2}\right)$ a cross disk and call the image (under the map $\pi$ ) of a pair of points (respectively arcs, disks) in $D_{1} \cup D_{2}$ a pair of points (respectively arcs, disks) in the cross disk, where $\pi: \widetilde{M} \rightarrow M$ is the covering map.

We denote by $\mathcal{F}$ the set of $\pi_{1}$-injective, $\partial$-injective and least weight surfaces in $M$ whose boundaries are embedded in $\partial M$. Let $\mathcal{F}_{R}=\{F \in \mathcal{F}$ : there are no cross disks of size $R$ in $\widetilde{F}$, where $\widetilde{F}$ is the preimage of $F$ in $\widetilde{M}$. The following lemma is due to Choi [5].

Lemma 3.2 There is a finite collection of immersed branched surfaces such that every surface in $\mathcal{F}_{R}$ is fully carried by an immersed branched surface in this collection.

Proof Let $T$ be a tetrahedron in the triangulation $\mathcal{T}$ of $M$ and $d_{i} \subset F \cap T$ be a normal disk $(i=1,2,3)$, where $F \in \mathcal{F}_{R}$. Suppose that $\widetilde{T}$ is a lift of $T$ in $\widetilde{M}, \widetilde{d}_{i}$ is a lift of $d_{i}$ in $\widetilde{T}$, and $F_{i}$ is the plane in $\widetilde{F}$ that contains $\widetilde{d}_{i}$ $(i=1,2,3)$, where $\widetilde{F}$ is the preimage of $F$ in $\widetilde{M}$. We call $D_{N}\left(d_{i}\right)=\left\{x \in F_{i}\right.$ : $d(x, p) \leq N$, where $\left.p \in \widetilde{d}_{i}\right\}$ a surface of radius $N$ with center $\widetilde{d}_{i}$. Note that, topologically, $D_{N}\left(d_{i}\right)$ may not be a disk under this discrete metric.

Next, we will define an equivalence relation. We say that $d_{1}$ is equivalent to $d_{2}$ if $D_{k R}\left(d_{1}\right)$ is parallel to $D_{k R}\left(d_{2}\right)$ and $F_{1} \cap F_{2}=\emptyset$ (or $F_{1}=F_{2}$ ), where $k$ is fixed. We assume that $k$ is so large that $D_{k R}\left(d_{i}\right)$ contains a subdisk of size $R$ whose center is $\widetilde{d}_{i}(i=1,2)$. Note that, since $M$ is compact and every plane in $\widetilde{F}$ has least weight, $k$ can be chosen to be independent of the choices of $F \in \mathcal{F}_{R}$ 
and the normal disk $d_{i} \subset F$, ie, $k$ depends only on $R$ and the triangulation of $M$. Suppose that there are three normal disks $d_{1}, d_{2}$ and $d_{3}$ in $F \cap T$ so that $d_{1}$ is equivalent to $d_{2}$ and $d_{2}$ is equivalent to $d_{3}$. Then $D_{k R}\left(d_{1}\right)$ is parallel to $D_{k R}\left(d_{3}\right)$ by definition. If $F_{1} \neq F_{3}$ and $F_{1} \cap F_{3} \neq \emptyset$, by the assumption on $k$, there is a cross disk of size $R$ that consists of two disks from $F_{1}$ and $F_{3}$. This contradicts the hypothesis that $F \in \mathcal{F}_{R}$. Thus $d_{1}$ is equivalent to $d_{3}$, and the equivalence relation is well-defined.

Since $M$ is compact, for any normal disk $d$ in $\widetilde{M}$, the number of nonparallel (embedded) normal surfaces of radius $k R$ (with center $d$ ) is bounded by a constant $C$ that depends only on $k R$ and the triangulation of $M$. As there are no cross disks of size $R$, if $D_{k R}\left(d_{1}\right)$ is parallel to $D_{k R}\left(d_{2}\right)$, then $d_{1}$ and $d_{2}$ must be equivalent. Thus, there are at most $C$ equivalence classes among the normal disks of $F \cap T$ with the same disk type, and hence we can divide the disks in $F \cap T$ for each $T$ into at most $7 C$ equivalence classes, since there are 7 different types of normal disks in a tetrahedron. For any tetrahedron $T$, suppose there are $C_{T}\left(C_{T} \leq 7 C\right)$ equivalence classes in $F \cap T$. We put $C_{T}$ products $D_{i} \times I$ $\left(i=1, \ldots, C_{T}\right)$ in $T$ such that $D_{i} \times\{t\}$ is a normal disk and the normal disks of $F \cap T$ in the same equivalence class lie in the same product $D_{i} \times I$. Along $\mathcal{T}^{(2)}$, we can glue these products $D_{i} \times I$ 's together according to the equivalence classes, as in the construction of embedded branched surfaces in [9]. In fact, we can abstractly construct a branched surface $B$ and a map $f: N(B) \rightarrow M$ such that, for any tetrahedron $T, f\left(\partial_{v} N(B)\right) \subset \mathcal{T}^{(2)}$ and $f\left(N(B)-p^{-1}(L)\right) \cap T$ is exactly the union of the products $\operatorname{int}\left(D_{i}\right) \times I$ 's in $T$, where $L$ is the branch locus of $B, p: N(B) \rightarrow B$ is the map that collapses every interval fiber of $N(B)$ to a point, and $\operatorname{int}\left(D_{i}\right)$ denotes the interior of $D_{i}$. By our construction, $B$ does not contain a local picture like that in Figure 2.3, and hence it can be embedded in some 3-manifold [6]. Since the number of equivalence classes is bounded by a constant, there are only finitely many such immersed branched surfaces that fully carry surfaces in $\mathcal{F}_{R}$.

Corollary 3.3 Suppose $M$ is a compact, orientable, irreducible 3-manifold whose boundary is an incompressible torus. Then the surfaces in $\mathcal{F}_{R}$ can realize only finitely many slopes.

Proof Suppose that $F_{1}, F_{2} \in \mathcal{F}_{R}$ are fully carried by the same immersed branched surface $f: B \rightarrow M$. To simplify notation, we will also denote by $f$ the corresponding map from $N(B)$ to $M$. Since the surfaces in $\mathcal{F}_{R}$ are embedded along their boundaries, after some normal homotopy if necessary, we can assume that $\left.f\right|_{\partial B}$ is an embedding. Since the surfaces in $\mathcal{F}_{R}$ are $\pi_{1-}$ injective, the horizontal boundary of $f(N(B)) \cap \partial M$ does not contain any trivial 
circle component. Because of Lemma 3.2 and Proposition 2.5, we only need to show that $f(\partial B)$ does not bound a monogon in $\partial M$. We will show next that the existence of a monogon in $\partial M$ contradicts our assumption that our immersed surfaces have least weight. The proof is essentially the same as an argument in [9] for embedded branched surfaces.

Since $\left.f\right|_{\partial B}$ is an embedding, to simplify notation, we do not distinguish $\partial B$ and $f(\partial B)$, and denote $f(N(\partial B)$ ) by $N(\partial B)$, where $N(\partial B)$ is a fibered neighborhood of the train track $\partial B$. By our definition of immersed branched surface, we can assume that $F_{1} \subset f(N(B))$ and $f^{-1}\left(F_{1}\right)$ is an embedded surface fully carried by $N(B)$.

Suppose that $D \subset \partial M$ is a monogon, ie, $\partial D=\alpha \cup \beta$, where $\alpha$ is a vertical arc of $\partial_{v} N(\partial B)$ and $\beta \subset \partial_{h} N(\partial B)$. The component of $f\left(\partial_{v} N(B)\right)$ that contains $\alpha$ is a rectangle $E$ whose boundary consists of two vertical arcs $\alpha, \alpha^{\prime}$ in $\partial M$ and two arcs $\gamma, \gamma^{\prime}$ in $f\left(\partial_{v} N(B) \cap \partial_{h} N(B)\right)$. Since $F_{1}$ is fully carried by $f: B \rightarrow M$, after some normal homotopy, we may assume that $E$ is embedded, $\partial_{h} N(\partial B) \subset \partial F_{1}$, and $\gamma \cup \gamma^{\prime} \subset F_{1}$. Then $\delta=\beta \cup \gamma \cup \gamma^{\prime}$ is an arc in $F_{1}$ with $\partial \delta \subset \partial F_{1} \subset \partial M$, and $\delta$ can be homotoped rel $\partial \delta$ into $\partial M$. Since $F_{1}$ is $\partial$-injective, $\delta$ must be $\partial$-parallel in $F_{1}$. So, there is an arc $\delta^{\prime} \subset \partial F_{1}$ such that $\delta \cup \delta^{\prime}$ is a closed trivial curve in $F_{1}$. Suppose $\delta \cup \delta^{\prime}$ bounds a disk $\Delta$ in $F_{1}$, which may not be embedded. Moreover, $\alpha^{\prime} \cup \delta^{\prime}$ also bounds a disk $D^{\prime}$ in $\partial M$, since $\alpha^{\prime} \cup \delta^{\prime}$ forms a homotopically trivial curve in $M$. So, $D \cup E \cup \Delta \cup D^{\prime}$ forms an immersed sphere in $M$. Since $\pi_{2}(M)$ is trivial, we can homotope the sphere $D \cup E \cup \Delta \cup D^{\prime}$ (fixing $E$ ) into $E$. After this homotopy, we get an immersed surface in the same homotopy class as $F_{1}$ with less weight. This contradicts our least weight assumption on the surface $F_{1}$.

So, $\partial B$ does not bound any monogon. By Proposition $2.5, \partial F_{1}$ and $\partial F_{2}$ must have the same slope, and the corollary follows from Lemma 3.2.

\section{Limits of cross disks}

Let $\mathcal{H}$ be the set of injective and least weight surfaces with the 4-plane property in $M$. If there is a number $R \in \mathbb{R}$ such that $\mathcal{H} \subset \mathcal{F}_{R}$, by Corollary 3.3, the surfaces in $\mathcal{H}$ can realize only finitely many slopes. Suppose no such a number $R$ exists. Then there must be a sequence of surfaces $F_{1}, F_{2}, \ldots, F_{n}, \cdots \in \mathcal{H}$ such that, in the preimage of $F_{i}$ in $\widetilde{M}$ (denoted by $\widetilde{F}_{i}$ ), there is a cross disk $D_{i}=D_{i}^{\prime} \cup D_{i}^{\prime \prime}$ of size at least $i$, where $i \in \mathbb{N}$. Since $M$ is compact, after passing to a subsequence if necessary, we can assume that $D_{i}^{\prime}$ is parallel to a subdisk 
$\Delta_{i}$ of $D_{i+1}^{\prime}$ and $d\left(\partial \Delta_{i}-\partial \widetilde{M}, \partial D_{i+1}^{\prime}-\partial \widetilde{M}\right) \geq 1$, where $d(x, y)$ denotes the distance. We also assume that $\partial D_{i}^{\prime}$ lies in the 2 -skeleton.

Proposition 4.1 The intersection of $\pi\left(D_{i}\right)$ with any tetrahedron does not contain two quadrilateral normal disks of different types, where $\pi: \widetilde{M} \rightarrow M$ is the covering map.

Proof We know that any two quadrilateral normal disks of different types must intersect each other. Suppose that the intersection of $\pi\left(D_{i}\right)$ with a tetrahedron contains two different types of quadrilateral normal disks. Let $T$ be a lift of this tetrahedron in $\widetilde{M}$. Then, in each of the two quadrilateral disk types, there is a pair of parallel normal disks in $\widetilde{F}_{i} \cap T$ that belong to different components of a cross disk. By the definition of cross disk, the two planes in $\widetilde{F}_{i}$ that contain the two parallel quadrilateral normal disks must intersect each other. Hence, the two different quadrilateral disk types give rise to 4 planes in $\widetilde{F}_{i}$ intersecting each other. Note that, these 4 planes are different planes in $\widetilde{F}_{i}$, since each plane is embedded in $\widetilde{M}$ by our assumptions. This contradicts the 4 -plane property.

Thus, as in [9], we can construct an embedded branched surface $B_{i}$ in $M$ such that $\pi\left(D_{i}\right)$ lies in $N\left(B_{i}\right)$ transversely intersecting every interval fiber of $N\left(B_{i}\right)$. In fact, for each normal disk type of $\pi\left(D_{i}\right) \cap T$, we construct a product $\delta \times I$, where $T$ is a tetrahedron and $\delta \times\{t\}$ is a normal disk of this disk type $(t \in I)$. Then, by Proposition 4.1, we can glue these products along $\mathcal{T}^{(2)}$ naturally to get a fibered neighborhood of an embedded branched surface $B_{i}$, and $\pi\left(D_{i}\right)$ can be isotoped into $N\left(B_{i}\right)$ transversely intersecting every interval fiber of $N\left(B_{i}\right)$. Note that $B_{i}$ may have nontrivial boundary. After some isotopy, we can assume that $\partial_{v} N\left(B_{i}\right) \cap \mathcal{T}^{(1)}=\emptyset$ and $N\left(B_{i}\right) \cap \mathcal{T}^{(2)}$ is a union of interval fibers of $N\left(B_{i}\right)$. By the definition of cross disk, we can also assume that every pair of points in the cross disk lies in the same $I$-fiber of $N\left(B_{i}\right)$.

Proposition 4.2 $N\left(B_{i}\right)$ can be split into an $I$-bundle over a compact surface such that, after normal homotopies, $\pi\left(D_{i}\right)$ lies in this $I$-bundle, transversely intersects its $I$-fibers, and every pair of points in the cross disk $\pi\left(D_{i}\right)$ lies in the same $I$-fiber of this $I$-bundle.

Proof By our construction above, $N\left(B_{i}\right) \cap \mathcal{T}^{(2)}$, when restricted to a 2 -simplex in $\mathcal{T}^{(2)}$, is a fibered neighborhood of a union of train tracks. Suppose that $\partial_{v} N\left(B_{i}\right)$ transversely intersects $\mathcal{T}^{(2)}$. First, we split $N\left(B_{i}\right)$ near $N\left(B_{i}\right) \cap \mathcal{T}^{(2)}$ to eliminate $\partial_{v} N\left(B_{i}\right) \cap \mathcal{T}^{(2)}$. 
Let $\Delta$ be a 2-simplex in $\mathcal{T}^{(2)}, \delta$ be a component of $\partial_{v} N\left(B_{i}\right) \cap \Delta$ and $N(\tau)$ be the component of $N\left(B_{i}\right) \cap \Delta$ that contains $\delta$. We associate every such component $\delta$ of $\partial_{v} N\left(B_{i}\right) \cap \Delta$ with a direction (in $\Delta$ ) that is orthogonal to $\delta$ and points into the interior of $N\left(B_{i}\right) \cap \Delta$. Let $V$ be the union of the interval fibers of $N(\tau)$ that contain some component of $\partial_{v} N\left(B_{i}\right) \cap \Delta$. After performing some isotopies, we can assume that every interval fiber in $V$ contains only one component of $\partial_{v} N\left(B_{i}\right) \cap \Delta$. We give every interval fiber in $V$ a direction induced from the direction of $\partial_{v} N\left(B_{i}\right) \cap \Delta$ defined above. Now $N(\tau)-V$ is a union of rectangles with two horizontal edges from $\partial_{h} N\left(B_{i}\right)$ and two vertical edges from $V$ or $\mathcal{T}^{(1)}$. Every vertical edge from $V$ has an induced direction.

Case 1 For any rectangle of $N(\tau)-V$, the direction of at most one vertical edge points inwards.

In this case, there is no ambiguity about the splitting near the rectangle. We split $N(\tau)$ as shown in Figure 4.1, pushing a component of $\partial_{v} N(B)$ across an edge of $\Delta$. During the splitting we may also push some double curves of $F_{i}$ across this edge. The effect of the splitting on $\pi\left(D_{i}\right)$ is just an isotopy. Thus, we can assume that any pair of points in the cross disk lies in the same interval fiber of the fibered neighborhood of the branched surface after this splitting.

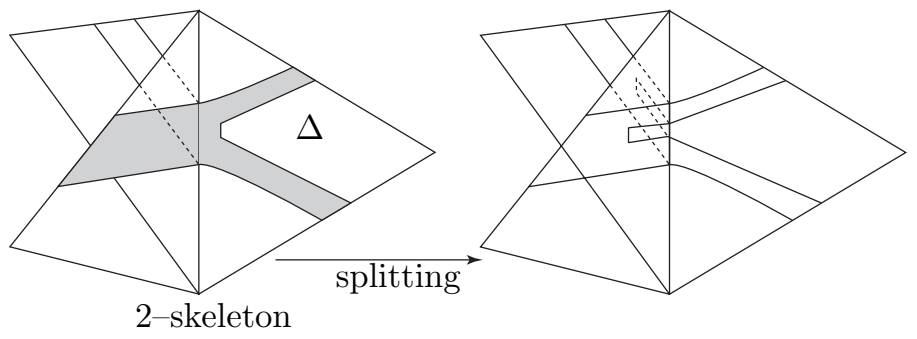

Figure 4.1

Case 2 There is a rectangle in $N(\tau)-V$ such that the directions of both vertical edges point inwards.

The local picture of such a rectangle must be as in Figure 4.2 (a), and there are (locally) three different splittings as shown in Figure 4.2 (b). We denote the rectangle by $R$ and the part of $N(\tau)$ as in Figure 4.2 (a) by $N(\tau)_{R}$. Then $N(\tau)_{R}-R$ consists of 4 components, and we call them UL (upper left) end, LL (lower left) end, UR (upper right) end and LR (lower right) end, as shown in Figure 4.2 (a). The intersection of $N(\tau)_{R}$ and the cross disk, ie, $\pi\left(D_{i}\right) \cap N(\tau)_{R}$, consists of arcs connecting the ends on the left side to the ends on the right side. An arc in $\pi\left(D_{i}\right) \cap N(\tau)_{R}$ is called a diagonal arc if it connects an upper end to a lower end. 


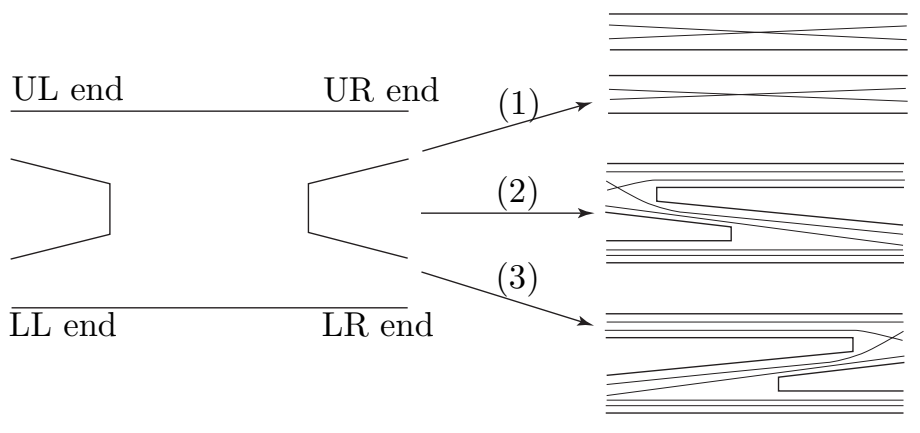

(a)

(b)

Figure 4.2

Claim $\pi\left(D_{i}\right) \cap N(\tau)_{R}$ does not contain two diagonal arcs, say $\alpha$ and $\beta$, such that $\alpha$ connects the UL end to the LR end, and $\beta$ connects the LL end to the UR end.

Proof of the claim Suppose that it contains such $\operatorname{arcs} \alpha$ and $\beta$. Then there is another arc $\alpha^{\prime}$ (respectively $\beta^{\prime}$ ) such that $\alpha \cup \alpha^{\prime}$ (respectively $\beta \cup \beta^{\prime}$ ) is a pair of arcs in the cross disk. So, $\alpha^{\prime}$ (respectively $\beta^{\prime}$ ) also connects the UL end to the LR end (respectively the LL end to the UR end). Note that $\alpha$ (or $\alpha^{\prime}$ ) and $\beta$ (or $\beta^{\prime}$ ) must have nontrivial intersection in $N(\tau)_{R}$. Next we consider a lift of $N(\tau)_{R}$ in $\widetilde{M}$ and still use the same notation. By the definition of cross disk, the 4 planes in $\widetilde{F}_{i}$ that contain $\alpha, \alpha^{\prime}, \beta$ and $\beta^{\prime}$ respectively must intersect each other in $\widetilde{M}$. Since every plane in $\widetilde{F}_{i}$ is embedded in $\widetilde{M}$, each is a different plane in $\widetilde{F}_{i}$. This contradicts the assumption that $F_{i}$ has the 4 -plane property.

Now we split $N\left(B_{i}\right)$ near $N(\tau)_{R}$ as follows. If there are no diagonal arcs in $\pi\left(D_{i}\right) \cap N(\tau)_{R}$, we split $N\left(B_{i}\right)$ in a small neighborhood of $N(\tau)_{R}$ as the splitting (1) in Figure 4.2. If there are diagonal arcs, we split it as the splitting (2) or (3) in Figure 4.2 according to the type of the diagonal arcs. Note that by the claim, diagonal arcs of different types cannot appear in $N(\tau)_{R}$ at the same time. As in case 1, we can assume that any pair of points of the cross disk lies in the same $I$-fiber after the splitting. To simplify the notation, we will also denote the branched surface after the splitting by $B_{i}$. Since $D_{i}$ is compact, after finitely many such splittings, $\partial_{v} N\left(B_{i}\right) \cap \mathcal{T}^{(2)}=\emptyset$. Now $\partial_{v} N\left(B_{i}\right)$ is contained in the interior of the 3-simplices, in other words, in a collection of disjoint open 3 -balls. So, every component of $\partial_{v} N\left(B_{i}\right)$ bounds a disk of contact. After we 
cut $N\left(B_{i}\right)$ along these disks of contact, as in [9], $\partial_{v} N\left(B_{i}\right)=\emptyset$ and $N\left(B_{i}\right)$ becomes an $I$-bundle over a compact surface. As before, we can assume that, after isotopies if necessary, every pair of points in the cross disk lies in the same $I$-fiber.

In the splittings above, we can preserve the intersection pattern of $\widetilde{F}_{i}$. For any arc $\gamma \subset F_{i} \cap \Delta$, since every arc in $F_{i} \cap \Delta$ is a normal arc in the triangle $\Delta$, we can assume that if an arc in $F_{i} \cap \Delta$ does not intersect $\gamma$ before the splitting, it does not intersect $\gamma$ after the splitting. Moreover, since the intersection of $F_{i}$ with any tetrahedron is a union of normal disks, we can assume that cutting the disks of contact does not destroy the 4 -plane property. The effect of the splitting on $F_{i}$ is just a normal homotopy pushing some double curves out of the cross disk. So, after the splitting, $F_{i}$ still satisfies the 4 -plane property and has least weight. Therefore, we can assume for each $i, \pi\left(D_{i}\right)$ lies in such an $I$-bundle over a compact surface and is transverse to the $I$-fibers. We will still denote this $I$-bundle by $N\left(B_{i}\right)$.

After collapsing every $I$-fiber of $N\left(B_{i}\right)$ to a point, we get a piece of embedded normal surface, which we denote by $S_{i}$, in $M$. Furthermore, $D_{i}^{\prime}$ is parallel to a subsurface of a component of $\widetilde{S}_{i}$, where $\widetilde{S}_{i}$ is the preimage of $S_{i}$ in $\widetilde{M}$.

There are only finitely many possible embedded normal surfaces (up to normal isotopy) in $M$ that are images (under the covering map $\pi$ ) of normal disks that are parallel to $D_{i}^{\prime}$. So, after passing to a subsequence and doing some isotopies if necessary, we can assume that $S_{i}$ is a subsurface of $S_{i+1}$. By our assumption $d\left(\partial D_{i}-\partial \widetilde{M}, \partial D_{i+1}-\partial \widetilde{M}\right) \geq 1$, we can consider the direct limit of the sequence $\left\{S_{i}\right\}$ as a (possibly noncompact) surface in $M$ whose boundary lies in $\partial M$, and its closure is a lamination in $M$. We can also consider this lamination as the inverse limit of a sequence of branched surfaces that carry $S_{i}$ (see [24] for details). We denote this lamination by $\lambda$. Since $\lambda$ is constructed using least weight disks, it is well known to experts that $\lambda$ is an essential lamination. We provide a proof below for completeness. Before we proceed, we will prove a useful lemma, which says that a monogon with a long (or large) "tail" does not exist.

Lemma 4.3 Let $F_{0}$ be a $\pi_{1}$-injective, $\partial$-injective least weight normal surface in a 3-manifold $M$ and $F$ be a plane in the preimage of $F_{0}$ in $\widetilde{M}$. Suppose that $F$ has least weight and there are two parallel disks $D_{1}$ and $D_{2}$ embedded in $F$. Suppose that there is a monogon, ie, an embedded disk $D$ with $\partial D=\alpha \cup \beta$, where $\beta=D \cap\left(F-\operatorname{int}\left(D_{1} \cup D_{2}\right)\right), \beta \cap D_{1}$ and $\beta \cap D_{2}$ are the two endpoints of $\alpha$, and $\alpha$ is an arc lying in a 2 -simplex. Then, weight $\left(D_{1}\right) \leq$ weight $(D)$. 
Proof As $D_{1}$ and $D_{2}$ are parallel, there is an embedded region $D^{2} \times[1,2]$ in $\widetilde{M}$, where $D^{2} \times\{t\}$ is parallel to $D_{1}$ for any $t \in[1,2]$ and $D^{2} \times\{i\}=D_{i}$ for $i=1,2$. Moreover, by our hypothesis on $\alpha$, we can assume that $\alpha=\{p\} \times[1,2]$, where $p \in \partial D^{2}$. After some isotopy, we can assume that $\left(\partial D^{2} \times[1,2]\right) \cap \widetilde{\mathcal{T}}^{(1)}=$ $\emptyset$, and hence the weight of $\partial D^{2} \times[1,2]$ is zero.

We take a parallel copy of the monogon $D$, say $D^{\prime}$. Let $\partial D^{\prime}=\alpha^{\prime} \cup \beta^{\prime}$ and $\alpha^{\prime}=\left\{p^{\prime}\right\} \times[1,2]\left(p^{\prime} \in \partial D^{2}\right)$, where $\alpha^{\prime}$ and $\beta^{\prime}$ are parallel and close to $\alpha$ and $\beta$ respectively. Then $\partial D^{2}-p \cup p^{\prime}$ consists of two $\operatorname{arcs} \gamma$ and $\eta$. By choosing $D^{\prime}$ to be close to $D$, we can assume that $\eta$ is the shorter one. The four $\operatorname{arcs} \beta, \beta^{\prime}$ and $\eta \times\{1,2\}$ form a circle that bounds a disk $\delta$ in $F$. We can assume that $D^{\prime}$ is so close to $D$ that the weight of $\delta$ is zero. $D_{1} \cup D_{2} \cup \delta$ is a disk in $F$ whose boundary is $\beta \cup \beta^{\prime} \cup(\gamma \times\{1,2\})$. The circle $\beta \cup \beta^{\prime} \cup(\gamma \times\{1,2\})$ also bounds another disk $D \cup D^{\prime} \cup(\gamma \times[1,2])$ in $\widetilde{M}$. Since $F$ has least weight, weight $\left(D_{1} \cup D_{2} \cup \delta\right)=$ 2 weight $\left(D_{1}\right) \leq$ weight $\left(D \cup D^{\prime} \cup \gamma \times[1,2]\right)=2$ weight $(D)+$ weight $(\gamma \times[1,2])$. By our assumption weight $(\gamma \times[1,2])=0$, we have weight $\left(D_{1}\right) \leq$ weight $(D)$.

We call a disk as the disk $D$ in the lemma above a monogon.

Lemma 4.4 The lamination $\lambda$ is an essential lamination.

Proof First we will show that every leaf of $\lambda$ is $\pi_{1}$-injective. Otherwise, there is a compressing disk $D$ embedded in $\widetilde{M}-\widetilde{\lambda}$ and $\partial D$ lies in a leaf $l$, where $\widetilde{\lambda}$ is the preimage of $\lambda$ in the universal cover $\widetilde{M}$. By our construction of $\lambda$, there is, for any $K>0$, a cross disk $D_{K}=D_{K}^{\prime} \cup D_{K}^{\prime \prime}$ of size at least $K$ that is parallel to a subsurface of $l$. Since $F_{K}$ is $\pi_{1}$-injective and has least weight, and since $\partial D$ is an essential curve in $l$, if $K$ is large, $D_{K}^{\prime}$ does not contain a closed curve that is parallel to $\partial D$. By choosing $K$ sufficiently large, we may assume that $D_{K}^{\prime}$ winds around $\partial D$ (in a small neighborhood of $D$ ) many times, as shown in Figure 4.3 (a). Let $N(D)$ be an embedded disk in $\widetilde{M}$ that contains $D$ in its interior, and $F$ be the plane in $\widetilde{F}_{K}$ that contains $D_{K}^{\prime}$. Since $F$ is embedded in $\widetilde{M}$, the component of $F \cap N(D)$ that contains the spiral arc in Figure 4.3 (a) must form a monogon with a long "tail" that consists of two parallel spiral arcs winding around $\partial D$ many times, as shown in Figure 4.3 (b). The weight of the monogon is at most weight $(D)$. If $K$ is large enough, the length of each spiral arc in the "tail" of the monogon is very large and, in a neighborhood of the "tail", we can choose two parallel disks with weight greater than weight $(D)$. This contradicts Lemma 4.3.

Next, we will show that every leaf of $\lambda$ is $\partial$-injective. Otherwise, there is a $\partial$-compressing disk $D^{\prime}$ whose boundary consists of two $\operatorname{arcs} \alpha$ and $\beta$, where 


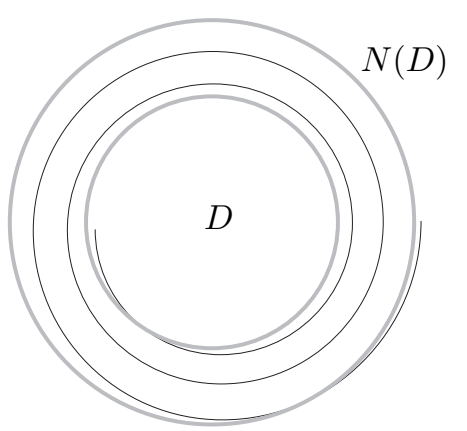

(a)

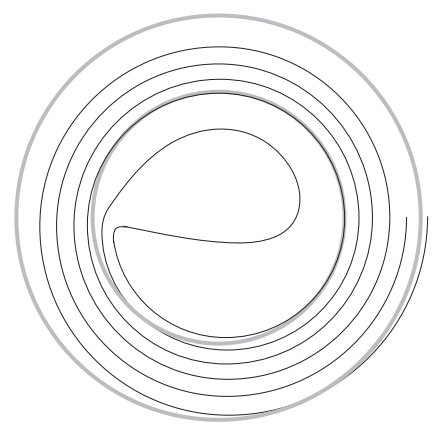

(b)

Figure 4.3

$\alpha \subset \partial M$ and $\beta$ is an essential arc in a leaf $l$. By our construction of $\lambda$, there is a cross disk $D_{n}=D_{n}^{\prime} \cup D_{n}^{\prime \prime}$ of size at least $n$ such that there are $\operatorname{arcs} \alpha_{n} \subset \partial M$ and $\beta_{n} \subset \pi\left(D_{n}^{\prime}\right)\left(\partial \alpha_{n}=\partial \beta_{n}\right)$ that are parallel and close to $\alpha$ and $\beta$ respectively. The two $\operatorname{arcs} \alpha_{n}$ and $\beta_{n}$ bound a disk $d_{n}$ that is parallel and close to $D^{\prime}$. Since the surface $F_{n}$ is $\partial$-injective, there must be an arc $\gamma_{n} \subset \partial F_{n}$ such that $\gamma_{n} \cup \beta_{n}$ bounds an immersed disk $\Delta_{n}$ in $F_{n}$. Since $\beta$ is an essential arc in $l$, by choosing $n$ sufficiently large, we can assume weight $\left(\Delta_{n}\right)>$ weight $\left(D^{\prime}\right)=$ weight $\left(d_{n}\right)$. Note that $\gamma_{n} \cup \alpha_{n}$ must bound a disk $\delta_{n}$ in $\partial M$ and that $d_{n} \cup \Delta_{n} \cup \delta_{n}$ is an immersed 2-sphere in $M$. Since $\pi_{2}(M)$ is trivial, we can homotope $\Delta_{n} \cup \delta_{n}$ to $d_{n}$ fixing $d_{n}$ and get another immersed surface $F_{n}^{\prime}$ that is homotopic to $F_{n}$. Moreover, weight $\left(F_{n}^{\prime}\right)-$ weight $\left(F_{n}\right)=$ weight $\left(d_{n}\right)-$ weight $\left(\Delta_{n}\right)<0$, which contradicts the assumption that $F_{n}$ has least weight.

It is easy to see from our construction that no leaf is a sphere or a disk, since the surfaces in the universal cover are embedded and are not spheres or disks. Also, if $\lambda$ is not end-incompressible, there must be a monogon with a long "tail", which contradicts Lemma 4.3 by the same argument as above. Therefore, $\lambda$ is an essential lamination.

\section{$5 \quad$ Measured sublaminations}

In this section, we will show that any minimal sublamination of $\lambda$ (constructed in section 4) has a transverse measure. A minimal lamination is a lamination that does not contain any proper sublamination. Using this result, we will prove Theorem 1, which can be viewed as a generalization of a theorem of Floyd and Oertel [9]. 
Let $\mu$ be a lamination in $M$ and $i: I \times I \rightarrow M$ be an immersion that is transverse to $\mu$, where $I=[0,1]$. We will call $\{p\} \times I$ a vertical arc, for any $p \in I$, and call $i(I \times I)$ a transverse rectangle if $i(I \times \partial I) \subset \mu$ and $i^{-1}(\mu)=I \times C$ for some closed set $C$ in $I$.

Lemma 5.1 Let $\mu$ be a minimal lamination. If $\mu$ has nontrivial holonomy, then there is a transverse rectangle $R: I \times I \rightarrow M$ such that $R(\{1\} \times I) \subset$ $R(\{0\} \times \operatorname{int}(I))$, where $\operatorname{int}(I)=(0,1)$.

Proof Since $\mu$ has nontrivial holonomy, there must be a map $g: S^{1} \times I \rightarrow M$, which is transverse to $\mu$, such that $g\left(S^{1} \times\{0\}\right) \subset L \subset \mu$ ( $L$ is a leaf) and $g^{-1}(\mu)$ consists of a collection of spirals and one circle $S^{1} \times\{0\}$ that is the limit circle of these spirals. Moreover, for any spiral leaf $l$ of $g^{-1}(\mu)$, there is an embedding $i$ : $[0, \infty) \times I \rightarrow S^{1} \times I$ such that $i^{-1}(l)=[0, \infty) \times\{1 / 2\}$ and $i([0, \infty) \times\{t\})$ is a spiral with limit circle $S^{1} \times\{0\}$ for each $t \in I$ (see the shaded region in Figure 5.1 (a)). Since $S^{1} \times\{0\}$ is the limit circle of $l$, for any arc $\{p\} \times[0, \epsilon] \subset S^{1} \times I$, there exists a number $N$, such that $i(\{N\} \times I) \subset\{p\} \times(0, \epsilon)$.

Since $\mu$ is a minimal lamination, every leaf is dense in $\mu$. Thus, there is a path $\rho: I \rightarrow L$ such that $\rho(0)=g(p, 0)$, where $p \in S^{1}$, and $\rho(1) \in g \circ i(\{0\} \times \operatorname{int}(I))$. Moreover, if $\epsilon$ is small enough, there is a transverse rectangle $r: I \times I \rightarrow M$ such that $\left.r\right|_{I \times\{0\}}=\rho, r(\{0\} \times I)=g(\{p\} \times[0, \epsilon])$, and $r(\{1\} \times I)=g \circ i(\{0\} \times$ $\left.\left[\delta_{1}, \delta_{2}\right]\right)$, where $\left[\delta_{1}, \delta_{2}\right] \subset I$. The concatenation of the transverse rectangle $r$ and $g \circ i\left([0, N] \times\left[\delta_{1}, \delta_{2}\right]\right)$, ie, $R: I \times I \rightarrow M$ where $R([0,1 / 2] \times I)=r(I \times I)$ and $R([1 / 2,1] \times I)=g \circ i\left([0, N] \times\left[\delta_{1}, \delta_{2}\right]\right)$, is a transverse rectangle we want, where $N$ is a number that $i(\{N\} \times I) \subset\{p\} \times(0, \epsilon) \subset S^{1} \times I$.

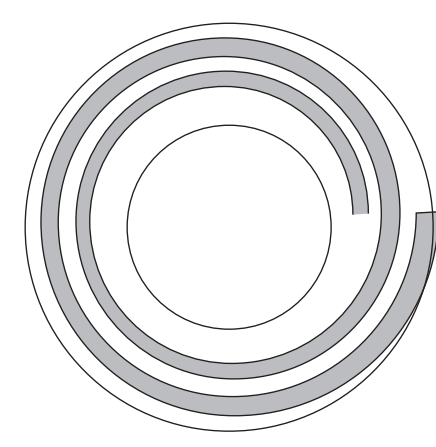

(a)

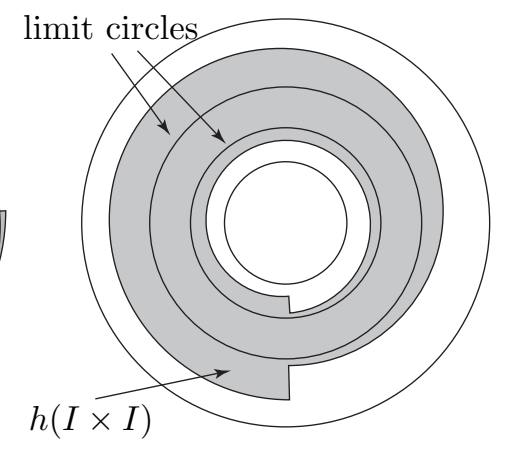

(b)

Figure 5.1 


\section{Remarks 5.2}

(1) The kind of construction in Lemma 5.1 was also used in [17].

(2) After connecting two copies of such transverse rectangles if necessary, we can assume that $R(\{1\} \times I) \subset R(\{0\} \times \operatorname{int}(I))$ in Lemma 5.1 preserves the orientation of the $I$-fibers. In other words, we may assume that there is a map $f: A \rightarrow M$ transverse to $\mu$, where $A=S^{1} \times I$, and an embedding (except for $\partial I \times I) h: I \times I \rightarrow A$, as shown in Figure 5.1 (b), such that $R=f \circ h$ and $f(A)$ lies in a small neighborhood of $R(I \times I)$.

(3) Let $f, h$, and $R$ be the maps above. Suppose that $L_{0}$ and $L_{1}$ are leaves in $\mu$ containing $R(I \times\{0\})$ and $R(I \times\{1\})$ respectively. Then $f^{-1}\left(L_{0} \cup L_{1}\right)$ contains two spirals of different directions whose limit circles are meridian circles of $A$ (see Figure 5.1 (b)). Note that $L_{0}$ and $L_{1}$ may be the same leaf and the two spirals may have the same limit circle.

(4) If $\mu$ is carried by a branched surface $B$, we can also assume that $R(\{q\} \times I)$ is a subarc of an interval fiber of $N(B)$ for any $q \in I$.

Lemma 5.3 Let $\lambda$ be the lamination constructed in section 4 and $\mu$ be any minimal sublamination of $\lambda$. Then $\mu$ has trivial holonomy.

Proof Suppose that $\mu$ has nontrivial holonomy. Since $\mu$ is a minimal lamination, by Remarks 5.2 above, there is an annulus $g: A=S^{1} \times I \rightarrow M$ such that $g^{-1}(\mu)$ contains two different kinds of spiral leaves, as shown in Figure 5.1 (b). From our construction of $\lambda$, there is a cross disk $D_{N}=D_{N}^{\prime} \cup D_{N}^{\prime \prime}$ such that $g^{-1}\left(\pi\left(D_{N}^{\prime}\right)\right)$ (respectively $g^{-1}\left(\pi\left(D_{N}^{\prime \prime}\right)\right)$ ) contains two arcs parallel and close to the two spirals respectively. We denote these two arcs by $\alpha_{0}^{\prime}$ and $\alpha_{1}^{\prime}$ (respectively $\alpha_{0}^{\prime \prime}$ and $\alpha_{1}^{\prime \prime}$ ), as shown in Figure 5.2 (a). Now we consider $g^{-1}\left(F_{N}\right)$, where $F_{N}$ is the corresponding least weight immersed surface with the 4-plane property. Since $F_{N}$ is compact, $g^{-1}\left(F_{N}\right)$ is compact. Denote the component of $g^{-1}\left(F_{N}\right)$ that contains $\alpha_{i}^{\prime}$ (respectively $\alpha_{i}^{\prime \prime}$ ) by $c_{i}^{\prime}$ (respectively $c_{i}^{\prime \prime}$ ), where $i=0,1$. Since $F_{N}$ is a normal surface, by Remarks 5.2 (4), we can assume that $g^{-1}\left(F_{N}\right)$ is transverse to each vertical arc $\{p\} \times I$ in $A$.

If $c_{1}^{\prime} \cap S^{1} \times\{0\}=\emptyset$, then $c_{1}^{\prime}$ is either a closed curve, as shown in Figure 5.2 (c), or an arc with both endpoints in $S^{1} \times\{1\}$, as shown in Figure 5.2 (b). Note that, by the Reeb stability theorem, any closed curve in a leaf with nontrivial holonomy must be an essential curve in this leaf. Since $\lambda$ is an essential lamination, $g\left(S^{1} \times\right.$ $\{0\})$ must be an essential curve in $M$, and we have the following commutative 


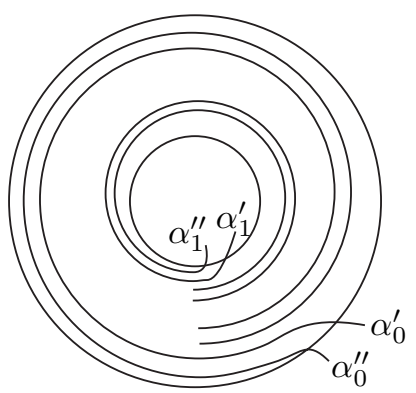

(a)

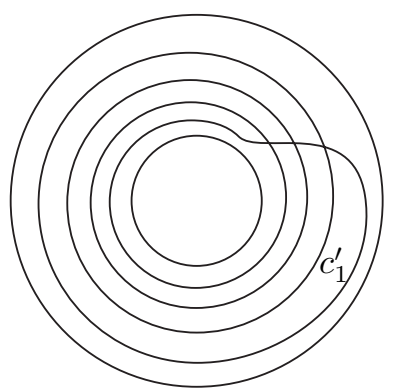

(c)

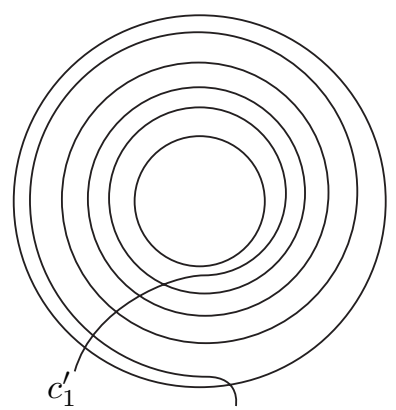

(b)

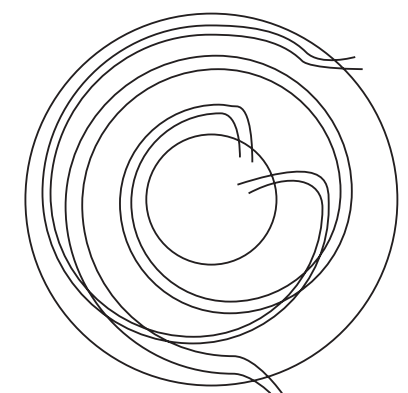

(d)

Figure 5.2

diagram, where $q$ is a covering map.

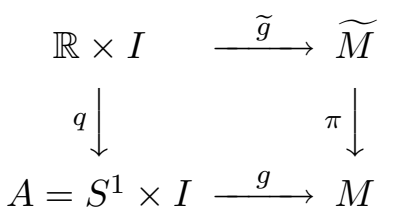

The pictures of $q^{-1}\left(c_{1}^{\prime}\right) \subset \widetilde{g}^{-1}\left(\widetilde{F}_{N}\right)$ are shown in Figure 5.3 (a) or (b) depending on whether $c_{1}^{\prime}$ is an arc with both endpoints in $S^{1} \times\{1\}$ or a closed curve. If $N$ is so large that $\alpha_{1}^{\prime}$ winds around $A$ more than four times, then there are four curves in $q^{-1}\left(c_{1}^{\prime}\right)$ intersecting each other, as shown in Figure 5.3 (a) and (b), which contradicts the assumption that $F_{N}$ has the 4 -plane property.

Thus, by the argument above, $c_{1}^{\prime}, c_{1}^{\prime \prime}, c_{0}^{\prime}$ and $c_{0}^{\prime \prime}$ must be arcs with endpoints in different components of $\partial A$, as shown in Figure $5.2(\mathrm{~d})$. In this case, $q^{-1}\left(c_{0}^{\prime} \cup\right.$ $\left.c_{1}^{\prime} \cup c_{0}^{\prime \prime} \cup c_{1}^{\prime \prime}\right)$ must contain $4 \operatorname{arcs} d_{0}^{\prime}, d_{0}^{\prime \prime}, d_{1}^{\prime}, d_{1}^{\prime \prime}$ as shown in Figure 5.3 (c), where $\widetilde{g}\left(d_{i}^{\prime} \cup d_{i}^{\prime \prime}\right)$ is the union of two arcs in different components of a cross disk 


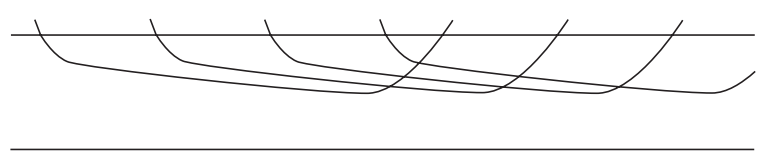

(a)

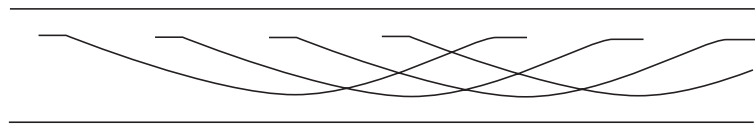

(b)

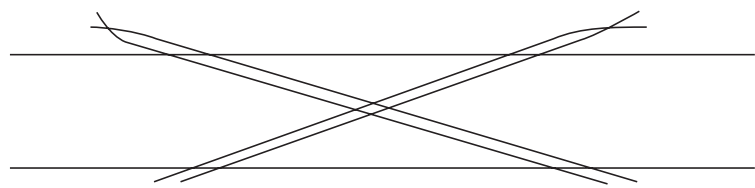

(c)

Figure 5.3

$(i=0,1)$. By the definition of cross disk, the 4 planes in $\widetilde{F}_{N}$ that contain $\widetilde{g}\left(d_{0}^{\prime}\right), \widetilde{g}\left(d_{0}^{\prime \prime}\right), \widetilde{g}\left(d_{1}^{\prime}\right)$ and $\widetilde{g}\left(d_{1}^{\prime \prime}\right)$ respectively must intersect each other, as shown in Figure 5.3 (c), which contradicts the assumption that $F_{N}$ has the 4-plane property.

The next theorem is a generalization of a theorem of Floyd and Oertel [9].

Theorem 1 Let $M$ be a closed, irreducible and non-Haken 3-manifold. Then there is a finite collection of immersed branched surfaces such that any surface in $M$ with the 4-plane property is fully carried by an immersed branched surface in this collection.

Proof If the set of immersed surfaces with the 4-plane property is a subset of $\mathcal{F}_{R}$ for some number $R$ (see section 3 for the definition of $\mathcal{F}_{R}$ ), then the theorem follows from by Lemma 3.2.

If there is no such a number $R$, by section 4 , there are a sequence of cross disks that give rise to an essential lamination $\lambda$. Let $\mu$ be a minimal sublamination of $\lambda$. Since $\mu$ is also an essential lamination, by [11], $\mu$ is fully carried by an embedded incompressible branched surface $B$. By Lemma 5.3, $\mu$ has no holonomy. A theorem of Candel [4] says that if a lamination has no holonomy then it has a transverse measure. So, $\mu$ has a transverse measure, and hence the system of the branch equations of $B$ (see [27]) has a positive solution. Since each 
branch equation is a linear homogeneous equation with integer coefficients, the system of branch equations of $B$ must have a positive integer solution. Every positive integer solution corresponds to an embedded surface fully carried by $B$. But, by a theorem of Floyd and Oertel [9], any surface fully carried by an incompressible branched surface must be incompressible. This contradicts the hypothesis that $M$ is non-Haken.

\section{Boundary curves}

Let $M$ be an irreducible 3-manifold whose boundary is an incompressible torus, $\lambda$ be the lamination constructed in section 4 and $\mu$ be a minimal sublamination of $\lambda$. Let $\left\{D_{i}=D_{i}^{\prime} \cup D_{i}^{\prime \prime}\right\}$ be the sequence of cross disks used in the construction of the lamination $\lambda$ in section 4 and let $F_{i}$ be the least weight immersed surface that contains $\pi\left(D_{i}\right)$. We denote the preimage of $F_{i}$ in $\widetilde{M}$ by $\widetilde{F}_{i}$. Suppose that $M$ does not contain any nonperipheral closed embedded incompressible surfaces.

Lemma $6.1 \mu \cap \partial M \neq \emptyset$

Proof Suppose that $\mu \cap \partial M=\emptyset$. Then $\mu$ is fully carried by an incompressible branched surface $B$ and $B \cap \partial M=\emptyset$. As in the proof of Theorem 1 (see section 5 ), the linear system of branch equations must have integer solutions that correspond to incompressible surfaces. Since $B \cap \partial M=\emptyset$ and $M$ does not contain any nonperipheral closed incompressible surfaces, those incompressible surfaces corresponding to the integer solutions must be $\partial$-parallel tori.

Let $N(B)$ be a fibered neighborhood of $B, C$ be the component of $M-$ $N(B)$ that contains $\partial M$, and $T_{1}, T_{2}, \ldots, T_{n}$ be a collection of $\partial$-parallel tori whose union corresponds to a positive integer solution of the system of branch equations. After isotopies, we can assume that every $T_{i}$ is transverse to the interval fibers of $N(B)$ and $\partial_{h} N(B) \subset \cup_{i=1}^{n} T_{i}$. Let $A$ be a component of $\partial_{h} N(B)$ that lies in the closure of $C$.

Claim The surface $A$ must be a torus.

Proof of the claim We first show that $A$ is not a disk. Suppose $A$ is a disk. Let $\nu$ be the component of $\partial_{v} N(B)$ that contains $\partial A$. Then $\partial \nu-\partial A$ is a circle in the boundary of a component $D$ of $\partial_{h} N(B)$. Since $\partial_{h} N(B)$ is incompressible and $A$ is a disk, $D$ must be a disk. So $A \cup \nu \cup D$ is a 2 -sphere. 
Since $M$ is irreducible, $A \cup \nu \cup D$ must bound a 3 -ball that contains $\cup_{i=1}^{n} T_{i}$, which contradicts the assumption that $T_{i}$ is incompressible.

If $\partial A=\emptyset$, since $\partial_{h} N(B) \subset \cup_{i=1}^{n} T_{i}, A$ is a $\partial$-parallel torus.

Suppose $\partial A \neq \emptyset$ and $A \subset T_{1}$. If there is a component of $\partial A$ that is a trivial circle in $T_{1}$ then, since $A$ is not a disk, there must be a trivial circle in $\partial A$ that bounds a disk in $T_{1}-A$. We can isotope this disk by fixing its boundary and pushing its interior into the interior of $N(B)$ so that it is still transverse to the $I$-fibers of $N(B)$, and we get a disk transverse to the $I$-fibers of $N(B)$ with its boundary in $\partial_{v} N(B)$. By definition, this is a disk of contact [9], which contradicts the assumption that $B$ is an incompressible branched surface. So, every circle of $\partial A$ must be an essential curve in $T_{1}$, and hence $A$ must be an annulus.

Let $c$ be a component of $\partial A, \nu^{\prime}$ be a component of $\partial_{v} N(B)$ that contains $c$, and $c^{\prime}=\partial \nu^{\prime}-c$ be the other boundary component of $\nu^{\prime}$. We denote the component of $\partial_{h} N(B)$ containing $c^{\prime}$ by $A^{\prime}$. By the argument above, $A^{\prime}$ must also be an annulus. If $A$ and $A^{\prime}$ belong to different tori, then $\nu^{\prime}$ is a vertical annulus in the product region $T^{2} \times I$ bounded by the two tori. This contradicts the assumptions that those tori are $\partial$-parallel and $\partial M \subset C$. Thus, $A$ and $A^{\prime}$ must belong to the same torus $T_{1}$. Then, $\nu^{\prime}$ must be an annulus in the $T^{2} \times I$ region bounded by $T_{1}$ and $\partial M$, and $\partial \nu^{\prime} \subset T_{1}$. So, the vertical arcs of $\nu^{\prime}$ can be homotoped rel $\partial \nu^{\prime}$ into $T_{1}$. This gives rise to a monogon and hence contradicts the assumption that $B$ is an incompressible branched surface [9]. Therefore, $\partial A=\emptyset$ and $A$ must be a torus.

By the claim and our assumptions, $C$ must be a product region $T^{2} \times I$ where $T^{2} \times\{1\}=\partial M$ and $T^{2} \times\{0\}=A \subset \partial_{h} N(B)$. Since $\mu$ is fully carried by $B$, we can assume that $A \subset \mu$ is a leaf. After choosing a sub cross disk if necessary, we can assume that there is a cross disk $D_{K}=D_{K}^{\prime} \cup D_{K}^{\prime \prime}$ of size at least $K$ such that $\pi\left(D_{K}^{\prime}\right)$ lies in a small neighborhood of $A$ that we denote by $T^{2} \times J$, where $J=[-\epsilon, \epsilon]$ and $A=T^{2} \times\{0\}$. By choosing $\epsilon$ small enough, we can assume $T^{2} \times\{t\}$ is a normal surface for any $t \in J$. Let $E$ be the component of $F_{K} \cap\left(T^{2} \times J\right)$ that contains $\pi\left(D_{K}^{\prime}\right)$ and $E^{\prime}$ be a component of the preimage of $E$ in $\widetilde{M}$. Let $F^{\prime}$ be the plane in $\widetilde{F}_{K}$ that contains $E^{\prime}$. So $E^{\prime}$ is embedded in a region $\mathbb{R}^{2} \times J$ in $\widetilde{M}, \partial E^{\prime} \subset \mathbb{R}^{2} \times\{ \pm \epsilon\}$. By choosing $\epsilon$ small enough and isotoping $F_{K}$, we can assume that $E^{\prime}$ is transverse to the $J$-fibers of $\mathbb{R}^{2} \times J$.

If $E^{\prime}$ is a compact disk, then $\partial E^{\prime}$ must be a circle in $\mathbb{R}^{2} \times\{ \pm \epsilon\}$ and $D_{K}$ must be in the region bounded by $\partial E^{\prime} \times J$. So, if $K$ is large, the disk in $\mathbb{R}^{2} \times\{ \pm \epsilon\}$ bounded by $\partial E^{\prime}$ is large. However, if the disk bounded by $\partial E^{\prime}$ is large enough, 
the 4 circles $g^{k}\left(\partial E^{\prime}\right)(k=0,1,2,3)$ must intersect each other, where $g$ is some element in $\pi_{1}(\partial M)$ that acts on $\widetilde{M}$ and fixes $\mathbb{R}^{2} \times J$. This violates the 4 -plane property, and hence $E^{\prime}$ cannot be a compact disk.

Suppose that $\widetilde{F}_{K} \cap\left(\mathbb{R}^{2} \times\{ \pm \epsilon\}\right)$ contains circular components. Let $e$ be an innermost such circle and $F_{e}$ be the plane in $\widetilde{F}_{K}$ that contains $e$. Then $e$ bounds a disk $D$ in $\mathbb{R}^{2} \times\{ \pm \epsilon\}$ and bounds another disk $D^{\prime}$ in $F_{e}$. We can assume that $D^{\prime} \cap \pi^{-1}\left(T^{2} \times\{ \pm \epsilon\}\right)=\partial D^{\prime}$; otherwise, we can choose $e$ to be a circle in $D^{\prime} \cap \pi^{-1}\left(T^{2} \times\{ \pm \epsilon\}\right)$ that is innermost in $D^{\prime}$. So, $D \cup D^{\prime}$ bounds a 3-ball in $\widetilde{M}$ and $\pi\left(D^{\prime}-\partial D^{\prime}\right) \cap\left(T^{2} \times J\right)=\emptyset$. Then, we can homotope $\pi\left(D^{\prime}\right)$ to $\pi(D)$ fixing $\pi(e)$. We denote by $F_{K}^{\prime}$ the surface after this homotopy and denote by $F_{e}^{\prime}$ the plane in $\widetilde{F}_{K}^{\prime}$ (the preimage of $F_{K}^{\prime}$ in $\widetilde{M}$ ) that contains $e$. Let $e^{\prime}$ be another component of $\pi^{-1}(\pi(e))$ and $F_{e^{\prime}}$ (respectively $F_{e^{\prime}}^{\prime}$ ) be the plane in $\widetilde{F}_{K}$ (respectively $\widetilde{F}_{K}^{\prime}$ ) that contains $e^{\prime}$. Since $D$ is innermost, if $F_{e} \cap F_{e^{\prime}}=\emptyset$, then $F_{e}^{\prime} \cap F_{e^{\prime}}^{\prime}=\emptyset$. Hence, $F_{K}^{\prime}$ is a surface homotopic to $F_{K}$ and $F_{K}^{\prime}$ also has the 4 -plane property. Note that since $F_{K}$ has least weight and $\mu$ is the "limit" of least weight cross disks, both $D$ and $D^{\prime}$ have least weight and weight $(D)=\operatorname{weight}\left(D^{\prime}\right)$. Thus, $F_{K}^{\prime}$ also has least weight and $F_{K}^{\prime} \cap T^{2} \times\{ \pm \epsilon\}$ has fewer trivial circles after a small homotopy. So, we can assume that $\widetilde{F}_{K} \cap \mathbb{R}^{2} \times\{ \pm \epsilon\}$ contains no trivial circles. Note that since $E^{\prime}$ can never be a compact disk by the argument above, this homotopy will not push the entire $E^{\prime}$ out of $\mathbb{R}^{2} \times J$. Therefore, we can assume that $E^{\prime}$ is a noncompact and simply connected surface.

If $\partial E^{\prime} \cap \mathbb{R}^{2} \times\{\epsilon\}$ has more than one component, then since we have assumed that $E^{\prime}$ is transverse to the $J$-fibers of $\mathbb{R}^{2} \times J, \partial E^{\prime} \cap \mathbb{R}^{2} \times\{\epsilon\}$ bounds a (noncompact) region $Q$ in $\mathbb{R}^{2} \times\{\epsilon\}, D_{K}^{\prime} \subset Q \times J$, and $\partial Q$ contains more than one line. Moreover, since $E^{\prime}$ is transverse to the $J$-fibers, it is easy to see that, for any element $g \in \pi_{1}(\partial M)$ that acts on $\widetilde{M}$ fixing $\mathbb{R}^{2} \times J$, if $Q \neq g(Q)$ and $Q \cap g(Q) \neq \emptyset$ in $\mathbb{R}^{2} \times\{\epsilon\}$, then $E^{\prime} \cap g\left(E^{\prime}\right) \neq \emptyset$. If $K$ is large, the distance between any two lines in $\partial Q$ must be large. Thus, by assuming $D_{K}^{\prime}$ to be large, we can always find a nontrivial element $g$ in $\pi_{1}(\partial M)$ such that the $g^{k}(Q)$ 's $(k=0,1,2,3)$, and hence the $g^{k}\left(E^{\prime}\right)$ 's $(k=0,1,2,3)$ intersect each other, which contradicts the 4 -plane property.

Therefore, $\partial E^{\prime} \cap \mathbb{R}^{2} \times\{\epsilon\}$ must be a single line, and hence $E$ must be an immersed annulus in $T^{2} \times J$ with one boundary component in $T^{2} \times\{\epsilon\}$ and the other boundary component in $T^{2} \times\{-\epsilon\}$. By our construction, weight $(E)$ is large if $K$ is large. We can always find an immersed annulus $A_{E} \subset T^{2} \times J$ with $\partial A_{E}=\partial E$ and weight $\left(A_{E}\right)$ relatively small. So, the surface $\left(F_{K}-E\right) \cup A_{E}$ is homotopic to $F_{K}$ and has less weight. The homotopy is like a Dehn twist 
unwrapping $E$ to get $A_{E}$. This contradicts the assumption that $F_{K}$ has least weight in its homotopy class. So, $\mu \cap \partial M$ cannot be empty.

Lemma 6.2 $\partial \mu$ is a lamination by circles.

Proof Since $\mu$ is a measured lamination and $\partial M$ is a torus, $\partial \mu$ is either a lamination by circles or a lamination by lines with an irrational slope. Suppose $\mu$ is fully carried by an incompressible branched surface $B$. Let $\mathcal{S}$ be the solution space of the system of branch equations of $B$. Since the coefficients of the branch equations are integers, there are finitely many positive integer solutions that generate $\mathcal{S}$, ie, any point (solution) in $\mathcal{S}$ can be written as a linear combination of these integer solutions. Every such integer solution gives rise to an incompressible surface fully carried by $B$. By Hatcher's theorem, these surfaces have the same boundary slope. The boundary slope of any measured lamination $\mu$ fully carried by $B$ is equal to the measure of a longitude of $\partial M$ divided by the measure of a meridian. Hence, the boundary slope can be expressed as a fraction with both numerator and denominator homogeneous linear functions of the weights of the branch sectors. Note that, similar to the proof of Hatcher's theorem, we can choose a transverse orientation for $\partial B$ and assume the homogeneous linear functions above are fixed in the calculation of the boundary slopes of any surfaces or measured laminations fully carried by $B$. Since the solution in $\mathcal{S}$ that corresponds to $\mu$ is a linear combination of those integer solutions, and since the boundary slopes of those integer solutions (plugging into the fraction described above) are the same, $\partial \mu$ must have the same slope as the boundary slope of these incompressible surfaces. Therefore, the boundary of any measured lamination fully carried by $B$ is a lamination by circles with the same slope.

Lemma 6.3 Let $\left\{D_{i}=D_{i}^{\prime} \cup D_{i}^{\prime \prime}\right\}$ be the sequence of cross disks used in the construction of an essential lamination in section 4 , and $F_{i}$ be the immersed surface with the 4-plane property that contains $\pi\left(D_{i}\right)$. Then, $\left\{F_{i}\right\}$ contains a subsequence of surfaces with the same boundary slope.

Proof Let $\lambda$ be the essential lamination constructed using $\left\{D_{i}\right\}$ as in section 4 , and $\mu$ be a minimal sublamination of $\lambda$. Then, by Lemma $6.2, \partial \mu$ is a lamination by circles. Let $B$ be an incompressible branched surface that fully carries $\mu$. Since $\partial \mu$ is a union of parallel circles, we can assume that $\partial B$ is a union of circles. Let $N(B)$ be a fibered neighborhood of $B, \widetilde{B}=\pi^{-1}(B)$ and $N(\widetilde{B})=\pi^{-1}(N(B))$. We can assume that each cross disk $D_{i}$ lies in $N(\widetilde{B})$, 
otherwise, we can choose a large sub cross disk of $D_{i}$ that lies in $N(\widetilde{B})$ for each $i$, and the proof is the same.

Suppose the lemma is not true, then we can choose $\left\{F_{i}\right\}$ to be a sequence of surfaces no two of which have the same boundary slopes. We can also assume that $\partial F_{k}$ has a different slope from $\partial \mu$ for each $k$. Then $\pi\left(D_{k}\right)$ is a piece of immersed surface in $N(B)$ transverse to every $I$-fiber, and $\pi\left(D_{k}\right) \cap \partial M$ is a union of spirals in $N(B) \cap \partial M$. We give each component of $\partial B$ an orientation so that they represent the same element in $H_{1}(\partial M)$. This orientation of $\partial B$ determines an orientation for each $I$-fiber of $N(B) \cap \partial M$. As in the proof of Hatcher's theorem, the orientation of the $I$-fibers and a normal direction of $\partial M$ uniquely determine an orientation for every curve in $N(B) \cap \partial M$ that is transverse to the $I$-fibers of $N(B)$.

Claim 1 If $k$ is sufficiently large, we can assume that each circle in $\partial F_{k}$ admits a direction along the curve that agrees with the induced orientation of every arc in $\partial F_{k} \cap N(B)$ described above.

Proof of claim 1 Suppose there is a circle in $\partial F_{k}$ that does not admit such an orientation. Then there must be a subarc $C$ of the circle outside $N(B) \cap \partial M$ connecting two spirals that are either in the same component of $N(B) \cap \partial M$, as shown in Figure 6.1 (a), or in different components of $N(B) \cap \partial M$ with incompatible induced orientations, as shown in Figure 6.1 (b). We will show that both cases contradict our assumption that $F_{k}$ is of least weight in its homotopy class. After assuming the size of the cross disk to be large, we can rule out the first possibility, ie, Figure 6.1 (a), by Lemma 4.3. To eliminate the second possibility, ie, Figure 6.1 (b), we use a certain triangulation of $M$ as follows.

By [19], there is a one-vertex triangulation $\mathcal{T}$ of $M$ and this vertex is in $\partial M$. Since $\partial M=T^{2}$, the induced triangulation of $\partial M$ must consist of two triangles as shown in Figure 6.2 (a). Now we glue a product region $T^{2} \times I(I=[0,1])$ to $M$ with $T^{2} \times\{0\}=\partial M$. Hence, $\left(\mathcal{T}^{(1)} \cap \partial M\right) \times I$ gives a cellulation of $T^{2} \times I$ that consists of a pair of triangular prisms. Then, we add a diagonal to each rectangular face of the prisms, which gives a triangulation of $T^{2} \times I$. Figure $6.2(\mathrm{~b})$ is a picture of the induced triangulation of a fundamental domain in the universal cover of $T^{2} \times I$. Since $M \cup\left(T^{2} \times I\right)$ is homeomorphic to $M$, we can assume that $M$ has a triangulation as that of $M \cup\left(T^{2} \times I\right)$ described above. To simplify notation, we still use $\mathcal{T}$ to denote this new triangulation of $M$. Now, $\mathcal{T}^{(0)} \cap \partial M$ is a single vertex $v$ and the intersection of its link hemisphere $H$ and $\mathcal{T}^{(1)}$ consists of 10 points of which 6 points lie in $\partial H \subset \partial M$. 


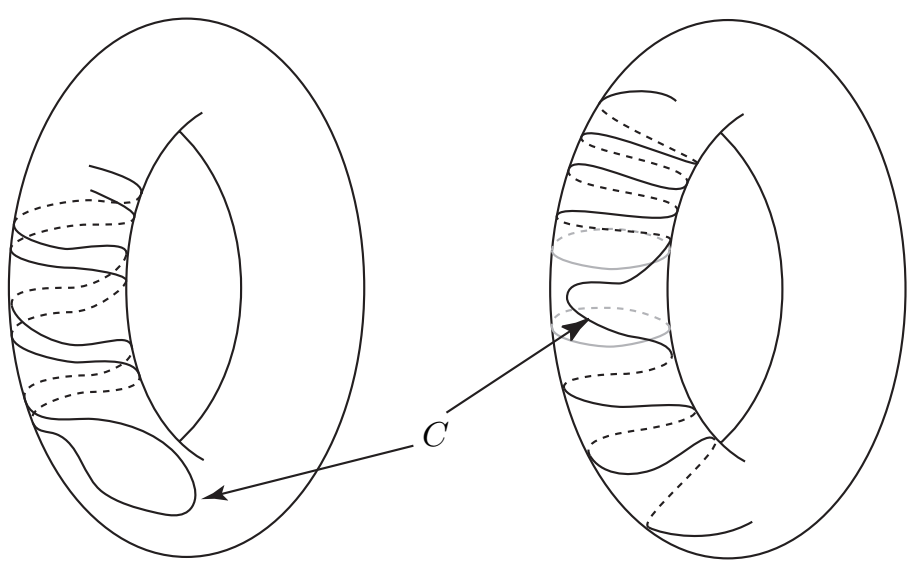

(a)

(b)

Figure 6.1

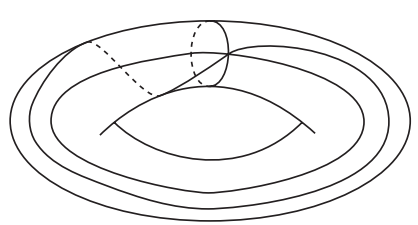

(a)

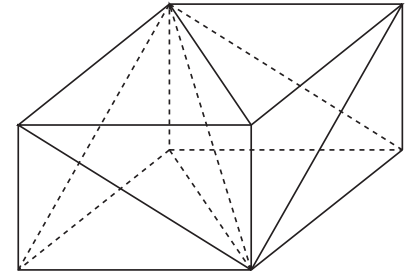

(b)

Figure 6.2

We assume that our immersed surfaces are normal and have least weight with respect to the triangulation above. Suppose the second case, ie, Figure 6.1 (b), occurs. Let $A$ be the annular component of $\partial M-N(B)$ that contains the arc $C$. Then we isotope $F_{k}$ by pushing $C$ along $A$ to "unwrap" the spirals in a small neighborhood of $\partial M$, as shown in Figure 6.3 (a) and (b). If the vertex $v$ is not in $A$, then after this isotopy, $\left|\partial F_{k} \cap \mathcal{T}^{(1)}\right|$ decreases and $\left|\left(F_{k}-\partial F_{k}\right) \cap \mathcal{T}^{(1)}\right|$ does not change. This contradicts the assumption that $F_{k}$ has least weight. So $v \in A$. If every edge of $\mathcal{T}^{(1)} \cap \partial M$ intersects $\partial A$ nontrivially, then after $C$ passes through the vertex $v$ during the isotopy, $\left|\partial F_{k} \cap \mathcal{T}^{(1)}\right|$ decreases by 6 and $\left|\left(F_{k}-\partial F_{k}\right) \cap \mathcal{T}^{(1)}\right|$ increases by 4 . Hence, the total weight of $F_{k}$ decreases, which also gives a contradiction. Therefore, there is an edge $e$ of $\mathcal{T}^{(1)} \cap \partial M$ lying inside $A$, as shown in Figure 6.3 (a). Then by our construction of the triangulation, $e$ forms a meridian circle of the annulus $A$ and there is at most 
one such edge. After $C$ passes through $v$ in the isotopy above, $\left|\partial F_{k} \cap \mathcal{T}^{(1)}\right|$ decreases by $4,\left|\left(F_{k}-\partial F_{k}\right) \cap \mathcal{T}^{(1)}\right|$ increases by 4 , and the total weight does not change.

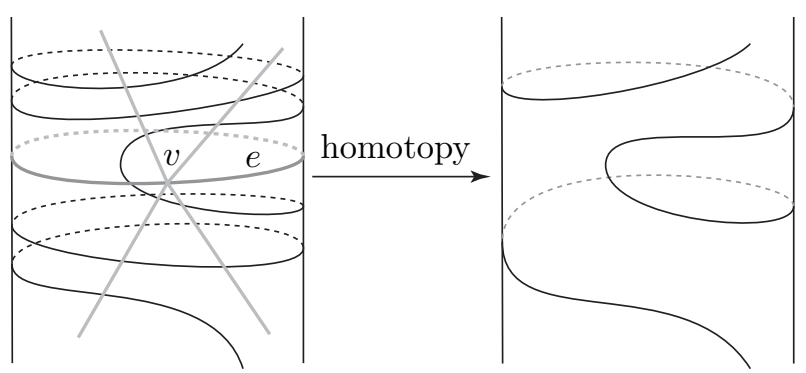

(a)

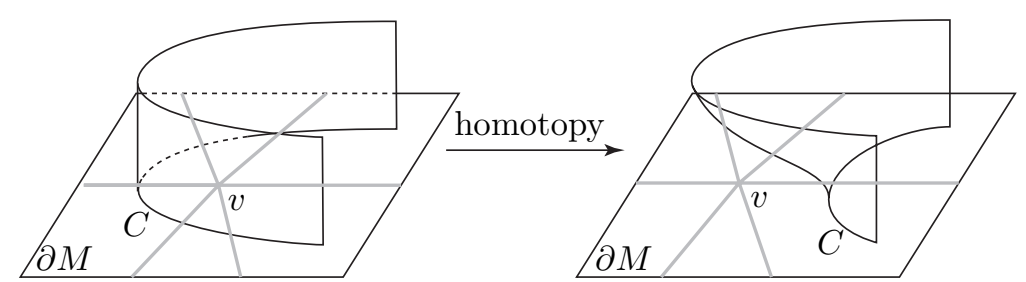

(b)

Figure 6.3

Now, we will see exactly what happens in a tetrahedron. Let $T$ be a tetrahedron with a face $\Delta$ in $\partial M$. There is a normal $\operatorname{arc} \delta$ in $C \cap \Delta$ that cuts off a subtriangle (in $\Delta \cap A$ ) that contains the vertex $v$. The normal disk of $F_{k} \cap T$ containing $\delta$ is either a triangle or a quadrilateral. If we do the isotopy as in Figure 6.3 (b) by pushing $C$ across $v$, then the effect of this isotopy on the normal disk that contains $\delta$ is either as in Figure 6.4 (a), in which case the normal disk is a triangle, or as in Figure 6.4 (b), in which case the normal disk is a quadrilateral. In the first case, as shown in Figure 6.4 (a), the disk is no longer a normal disk after the isotopy. So, we can perform another homotopy to make $F_{k}$ (after the first isotopy) a normal surface. This homotopy reduces $\left|F_{k} \cap \mathcal{T}^{(1)}\right|$ by at least 2 as we push the disk in Figure 6.4 (a) across the edge, which contradicts the assumption that $F_{k}$ has least weight. Thus, every normal disk that contains such an $\operatorname{arc} \delta$ is a quadrilateral. Since there are only two triangles in $\partial M$, and since the edge $e$ lies inside $A$, there must be two $\operatorname{arcs} \delta_{1}$ and $\delta_{2}$ in $C$ that cut off two corners of the same triangle (in the induced triangulation of 
$\partial M)$. By the argument above, the two normal disks that contain $\delta_{1}$ and $\delta_{2}$ respectively must be two quadrilaterals of different normal disk types in the same tetrahedron. Note that, during the isotopy as in Figure 6.3, we push parts of $\partial F_{k}$ from $N(B) \cap \partial M$ into the annulus $A$, and by unwrapping every such spiral, we can assume that any two parallel normal disks in $F_{k}$ remain parallel after the isotopies. We keep unwrapping the spirals by isotopies as in Figure 6.3. Either the weight of $F_{k}$ can be reduced at a certain stage, or we can eventually push parts of $\pi\left(D_{k}\right) \cap \partial M$ into the annulus $A$. In particular, after unwrapping the spirals enough times, we can assume that the $\delta_{1}$ and $\delta_{2}$ above lie in the cross disk. Then, we can assume that there is a pair of normal disks in the cross disk for each of the two quadrilateral normal disk types that correspond to the $\delta_{1}$ and $\delta_{2}$. Since any two quadrilateral normal disks of different types must intersect each other, those 4 quadrilaterals give rise to 4 planes in $\widetilde{F}_{k}$ intersecting each other (as in Proposition 4.1), which contradicts the hypothesis that $F_{k}$ has the 4 -plane property. So, if $k$ is large enough, we can reduce the weight of $F_{k}$ at a certain stage of the isotopy above. Therefore, Figure 6.1 (b) cannot occur and claim 1 holds.

The branched surface that fully carries $\mu$ also fully carries a compact surface, and by Lemma 6.2, the slope of $\partial \mu$ is the same as the boundary slope of an incompressible and $\partial$-incompressible surface. By Hatcher's theorem, there are only finitely many possible slopes for $\partial \mu$. If the lamination $\mu$ is constructed using cross disks from the sequence of surfaces $\left\{F_{k}\right\}$, then the arcs in $\partial F_{k}$ must wind around $\partial \mu$ many times (if $k$ is large). Therefore, by Corollary 3.3, our construction of $\mu$ and Claim 1 above, it is easy to see that there must be infinitely many slopes that cannot be the boundary slopes of surfaces with the 4-plane property, and Dehn fillings along these slopes yield 3-manifolds that cannot admit any nonpositive cubings (see the proof of Theorem 3 at the end of this paper). This can be viewed as a weaker version of Theorems 2 and 3 . To prove Theorem 2 to the full extent, which says that only finitely many slopes can be the boundary slopes of surfaces with the 4-plane property, we need to study the local pictures of the limit lamination and surfaces with the 4 -plane property in detail.

We first consider the case that $\mu$ is a compact orientable surface. The proof for the case that $\mu$ contains a noncompact leaf is similar. Let $\mu \times I \subset M$ $(I=[0,1])$ be a small neighborhood of $\mu$ in $M$, and $\widetilde{\mu} \times I$ be a component of the preimage of $\mu \times I$ in $\widetilde{M}$ with the induced fiber structure. Since $\mu$ is a compact embedded essential surface in $M, \widetilde{\mu} \times I$ can be considered as the universal cover of $\mu \times I$, and we can assume $\pi_{1}(\mu)$ acts on $\widetilde{M}$ fixing $\widetilde{\mu} \times I$. 


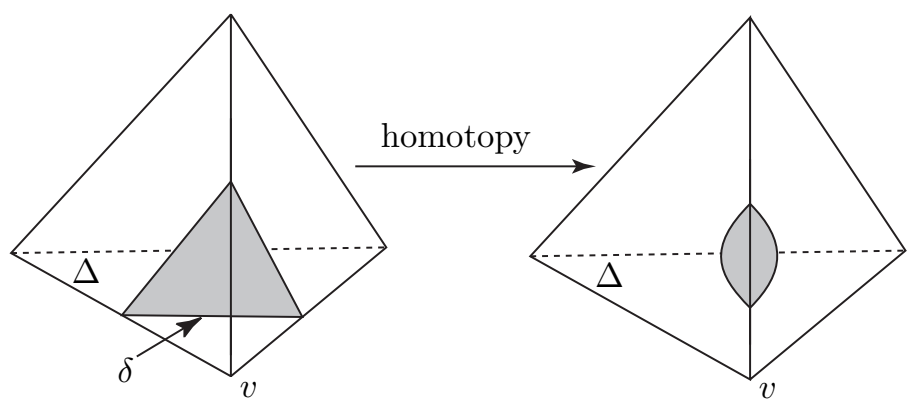

(a)

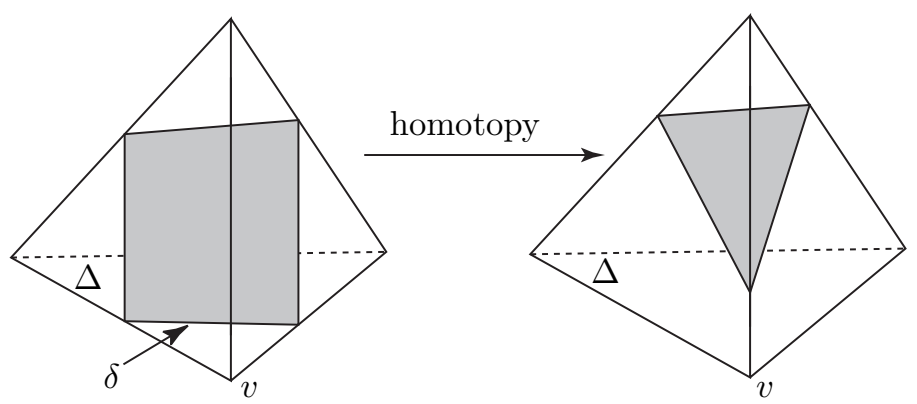

(b)

Figure 6.4

Suppose $k$ is large. By our construction of the lamination, there is always a large sub cross disk of $D_{k}=D_{k}^{\prime} \cup D_{k}^{\prime \prime}$ lying in $\widetilde{\mu} \times I$. To simplify notation, we assume that $D_{k} \subset \widetilde{\mu} \times I$; otherwise we use a large sub cross disk of $D_{k}$ and the proof is the same.

Let $F_{k}^{\prime}$ be the plane in $\widetilde{F}_{k}$ that contains $D_{k}^{\prime}, H^{\prime}=F_{k}^{\prime} \cap(\widetilde{\mu} \times I), H=\pi\left(H^{\prime}\right)$. Since we can give every component of $\partial F_{k}$ an orientation that agrees with the induced orientation of $\partial F_{k} \cap(\mu \times I)$ in claim 1, we can assume the sign of every intersection point of $\partial F_{k} \cap \partial S$ is always the same, where $S=\mu \times\{t\}(t \in I)$. Then, $H$ cannot be transverse to every $I$-fiber of $\mu \times I$, because otherwise, by the argument in the proof of Hatcher's theorem, $\partial F_{k}$ and $\partial S$ would have the same slope, which contradicts our assumptions. Figure 6.5 gives a local picture of $H$ where it is not transverse to an $I$-fiber of $\mu \times I$.

In fact, it is not hard to see that, in some tetrahedron $T$, there must be two different types of quadrilateral normal disks in $T \cap S$ and $T \cap F_{k}$ respectively. 
Otherwise, by an argument in [9], $H$ and $S$ lie in $N\left(B_{T}\right)$ and are transverse to the $I$-fibers of $N\left(B_{T}\right)$, where $N\left(B_{T}\right)$ is a fibered neighborhood of an embedded normal branched surface $B_{T}$. Hence, by the arguments in the proof of Hatcher's theorem, $F_{k}$ and $S$ have the same boundary slope (although $F_{k}$ is not embedded), which contradicts our assumption.

Since all these surfaces are normal, after a small homotopy, we can assume that each $I$-fiber of $\mu \times I$ either transversely intersects $H$ or entirely lies in $H$, in which case the local picture of this fiber is as shown in Figure 6.5, and we call such fibers puncturing fibers. We can assume $\partial F_{k} \cap(\partial \mu \times I)$ is a union of spirals, and by claim 1, the intersection points in $\partial F_{k} \cap \partial S(S=\mu \times\{t\})$ all have the same sign. Then, by our assumption on $H \cap(\mu \times I)$ and the argument above on Hatcher's trick, any arc of $F_{k} \cap S$ with endpoints in $\partial M$ must pass through a puncturing fiber. Since there is a large cross disk wrapping around the compact surface $\mu$ many times, such a puncturing fiber must puncture a cross disk, and we immediately get three planes (in the universal cover) intersecting each other. Moreover, any relatively short (compared with the size of the cross disk) arc with endpoints in different components of $\mu \times \partial I$ also punctures a cross disk. Furthermore, if we can find two such short arcs that are not far away from each other, then they puncture the same cross disk. If, in addition, the two planes that contain the two short arcs intersect each other, we get a contradiction to the 4 -plane property. This is the basic idea of our proof. After perturbing $F_{k}$ a little, we can assume that $F_{k} \cap(\mu \times I)$ is transverse to the $I$-fibers of $\mu \times I$ except at puncturing fibers and there are only finitely many puncturing fibers in $F_{k} \cap(\mu \times I)$.

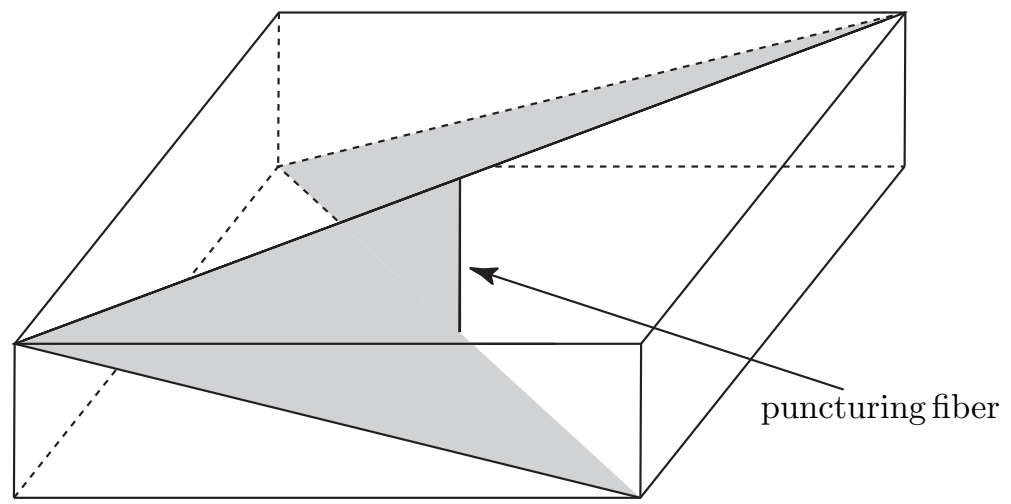

Figure 6.5

The following observation, which summarizes the argument above, is important to the remainder of the proof of Lemma 6.3. 
Observation 6.4 Let $\alpha_{i}(i=1,2)$ be an arc in $\widetilde{F}_{k} \cap(\widetilde{\mu} \times I)$ with two endpoints lying in different components of $\widetilde{\mu} \times \partial I$. Suppose length $\left(\alpha_{1}\right)$, length $\left(\alpha_{2}\right)$, and the distance between $\alpha_{1}$ and $\alpha_{2}$ are bounded by a fixed number. Then, if $k$ is large, $\alpha_{1}$ and $\alpha_{2}$ must puncture the same cross disk. Let $F^{(i)}$ denote the plane in $\widetilde{F}_{k}$ that contains $\alpha_{i}(i=1,2)$. If $F^{(1)} \cap F^{(2)} \neq \emptyset$ and $F^{(1)} \neq F^{(2)}, F^{(1)}$, $F^{(2)}$ and the two planes containing the two components of the cross disk are 4 planes in $\widetilde{F}_{k}$ intersecting each other, which contradicts the 4 -plane property.

We denote the puncturing fibers of $F_{k} \cap(\mu \times I)$ by $\gamma_{1}, \ldots, \gamma_{n}$. Let $q: \mu \times I \rightarrow \mu$ be the projection map. Hence, $q\left(\gamma_{1}\right), \ldots, q\left(\gamma_{n}\right)$ are points in $\mu$. Then, we can connect $q\left(\gamma_{1}\right), \ldots, q\left(\gamma_{n}\right)$ buy simple arcs $\alpha_{1}, \ldots, \alpha_{k}$ to form a 1-complex $\Gamma$ in which the $q\left(\gamma_{i}\right)$ 's are the $0-$ cells and the $\alpha_{i}$ 's are the $1-$ cells. Moreover, we can assume that $\mu-\Gamma$ is a union of disks and annular neighborhoods of $\partial \mu$. We denote the closure of the annular components of $\mu-\Gamma$ by $A_{1}, \ldots, A_{m}$, where $m$ is the number boundary components of $\mu$. Thus, for each $i$, one component of $\partial A_{i}$ is a boundary circle of $\mu$ and the other component of $\partial A_{i}$ lies in $\Gamma$. We denote $q^{-1}(\Gamma)$ and $q^{-1}\left(\alpha_{i}\right)$ by $\Gamma \times I$ and $\alpha_{i} \times I$ respectively, and denote the preimage of $\Gamma \times I$ in $\widetilde{\mu} \times I$ by $\widetilde{\Gamma} \times I$.

Let $S=\mu \times\{t\}$ and $\widetilde{S}=\widetilde{\mu} \times\{t\}(t \in I)$. We assume $S$ is transverse to $F_{k}$. As before, any double arc in $F_{k} \cap S$ or $\widetilde{F}_{k} \cap \widetilde{S}$ must pass through a puncturing fiber. Let $\beta$ be a subarc of a double arc of $\widetilde{F}_{k} \cap \widetilde{S}$ in $\widetilde{M}$ with one endpoint $Z_{0}$ in $\partial \widetilde{S} \cap \partial \widetilde{F}_{k}$ and the other endpoint in a puncturing fiber. We can assume the interior of $\beta$ does not intersect any puncturing fiber. We denote the closure of the component of $\widetilde{\mu} \times I-\widetilde{\Gamma} \times I$ containing $Z_{0}$ by $\widetilde{A}_{1} \times I$, and suppose $\pi\left(\widetilde{A}_{1} \times I\right)$ is $A_{1} \times I$ in $\mu \times I$ defined above, where $\pi: \widetilde{M} \rightarrow M$ is the covering map.

Let $D_{[k / 2]}=D_{[k / 2]}^{\prime} \cup D_{[k / 2]}^{\prime \prime}$ be a sub cross disk of $D_{k}=D_{k}^{\prime} \cup D_{k}^{\prime \prime}$ of size $[k / 2]$ and with the same center. By choosing an appropriate $t \in I$, we can assume that $Z_{0}$ lies in $D_{[k / 2]}^{\prime}$. Moreover, after some isotopy on $F_{k} \cap(\mu \times I)$ (or choosing an appropriate $\Gamma$ ), we can assume that there is a subarc of $\beta$, which we denote by $\beta_{0}$, properly embedded in $\widetilde{A}_{1} \times I$ such that $Z_{0} \in \partial \beta_{0}$ and $\beta_{0} \subset D_{[k / 2]}^{\prime}$. We denote the two lines in $\partial \widetilde{A}_{1}$ by $l_{1}$ and $l_{2}$, and suppose $Z_{0} \in l_{1} \times I$. Let $Z_{1}$ be the other endpoint of $\beta_{0}$. Hence, $Z_{1} \in l_{2} \times I \subset \widetilde{\mu} \times I$. By choosing $k$ to be large, we can assume the length of the curve in $\widetilde{F}_{k} \cap\left(l_{2} \times I\right)$ that contains $Z_{1}$ is large, since $Z_{1}$ lies in the sub cross disk $D_{[k / 2]}$.

We can assume that the interior of $\beta$ is transverse to $\widetilde{\Gamma} \times I$. Note that $\operatorname{int}(\beta)$ does not intersect any puncturing fiber in $\widetilde{\Gamma} \times I$. We denote the points in $\beta \cap(\widetilde{\Gamma} \times I)$ by $Z_{1}, \ldots, Z_{s}$, where $Z_{1}$ is as above and $Z_{s}=\partial \beta-Z_{0}$ lies in a 
puncturing fiber. These $Z_{i}$ 's divide $\beta$ into $s$ subarcs $\beta_{0}, \beta_{1}, \ldots, \beta_{s-1}$, where $\partial \beta_{i}=Z_{i} \cup Z_{i+1}$ and $\beta_{0}$ is as above.

We regard $\widetilde{\Gamma}$ as a 1 -complex in $\widetilde{\mu}$ with 0 -simplices corresponding to the puncturing fibers. Let $\alpha$ be any 1 -simplex in $\widetilde{\Gamma}$. So, $\alpha \times I \subset \widetilde{\Gamma} \times I$ is a vertical rectangle in $\widetilde{\mu} \times I$. We call an arc in $\widetilde{F}_{k} \cap(\alpha \times I)$ a $\partial$-parallel arc if the two endpoints of this arc lie in the same component of $\operatorname{int}(\alpha) \times \partial I$, where $\operatorname{int}(\alpha)$ denotes the interior of $\alpha$. We can perform some normal homotopy on $F_{k}$ to push all the $\partial$-parallel arcs out of $\pi(\alpha \times I)$, where $\pi: \widetilde{M} \rightarrow M$ is the covering map, so that if two arcs in $\widetilde{F}_{k} \cap(\alpha \times I)$ do not intersect each other, then after this homotopy, they do not intersect each other either. Hence, this normal homotopy preserve the 4 -plane property. Therefore, we can assume that $\widetilde{F}_{k} \cap(\alpha \times I)$ contains no $\partial$-parallel arcs for any 1 -simplex $\alpha$. Moreover, if $k$ is large, any $\partial$-parallel arc does not lie in the sub cross disk $D_{[k / 2]}$, and hence this homotopy does not affect the previous assumptions on $\beta_{0} \subset D_{[k / 2]}$.

Let $\zeta$ be an arc in $\widetilde{F}_{k} \cap(\alpha \times I)$. Since there is no $\partial$-parallel arc in $\widetilde{F}_{k} \cap(\alpha \times I)$, either the two endpoints of $\zeta$ lie in different components of $\operatorname{int}(\alpha) \times \partial I$, or one endpoint of $\zeta$ lies in a puncturing fiber $\gamma \subset \partial \alpha \times I$ in which case we denote the two planes in $\widetilde{F}_{k}$ containing $\zeta$ and $\gamma$ by $F_{\zeta}$ and $F_{\gamma}$ respectively. So, if the second case happens, either $F_{\zeta} \neq F_{\gamma}$ and $F_{\zeta} \cap F_{\gamma} \neq \emptyset$ or $F_{\zeta}=F_{\gamma}$. We call $\zeta$ a puncturing arc if either the two endpoints of $\zeta$ lie in different components of $\operatorname{int}(\alpha) \times \partial I$, or $F_{\zeta}=F_{\gamma}$. Thus, if $\zeta$ is a puncturing arc, there must be a relatively short arc in $F_{\zeta}$ containing $\zeta$ and with two endpoints in different components of $\widetilde{\mu} \times \partial I$. The role of a puncturing arc is the same as the role of a puncturing fiber, see Observation 6.4. Moreover, if $\zeta$ is not a puncturing arc, then one of the two puncturing fibers in $\partial \alpha \times I$ intersects the plane $F_{\zeta}$ nontrivially.

Claim 2 Each $Z_{i} \in \beta(1 \leq i \leq s-1)$ lies in a puncturing arc.

Proof of Claim 2 We first show that $Z_{s-1}$ lies in a puncturing arc, and then we inductively prove it for each $Z_{i}$. Suppose $Z_{s-1}$ lies in $\alpha \times I$, where $\alpha$ is a 1 -simplex of $\widetilde{\Gamma}$, and we denote the arc in $\widetilde{F}_{k} \cap(\alpha \times I)$ containing $Z_{s-1}$ by $\zeta_{s-1}$. Let $A \times I \subset \widetilde{\mu} \times I$ be the closure of the component of $\widetilde{\mu} \times I-\widetilde{\Gamma} \times I$ that contains $\beta_{s-1}$. So, $\beta_{s-1}$ is an properly embedded arc in $A \times I$ with $\partial \beta_{s-1} \subset \partial A \times I$. Since $\mu-\Gamma$ consists of disks and annular neighborhoods of circles in $\partial \mu$, either $A$ is a disk, or $A$ is of the form $[0,1] \times \mathbb{R}$ which can be considered as the universal cover of an annular neighborhood of a boundary circle of $\mu$.

If $A$ is a disk, since $\beta_{s-1}$ is properly embedded in $A \times I$, after some isotopy on $F_{k}$ if necessary, we can assume length $\left(\beta_{s-1}\right)$ is bounded by a number that depends 
only on $A$. If $\zeta_{s-1}$ is not a puncturing arc in $\alpha \times I$, since we have assumed that $\widetilde{F}_{k} \cap(\alpha \times I)$ does not contain any $\partial$-parallel arc, $\zeta_{s-1}$ must intersect at least one of the two puncturing fibers in $\partial \alpha \times I$. Let $\gamma$ be a puncturing fiber in $\partial \alpha \times I$ that intersects $\zeta_{s-1}$. By our definition of puncturing arc, the two planes in $\widetilde{F}_{k}$ containing $\gamma$ and $\beta_{s-1}$ intersect each other. By our construction above, $Z_{s} \in \partial \beta_{s-1}$ lies in another puncturing fiber, say $\gamma_{s}$. Since $A$ is a disk, the distance between the two puncturing fibers $\gamma$ and $\gamma_{s}$ is bounded by the diameter of the disk $A$. So, $\gamma$ and $\gamma_{s}$ puncture the same cross disk. Moreover, the plane containing $\gamma_{s}, \beta_{s-1}$ and $\zeta_{s-1}$ and the plane containing $\gamma$ intersect each other, which contradicts the 4-plane property as in Observation 6.4.

So, we only need to consider the case that $A$ is of the form $[0,1] \times \mathbb{R}$. We denote the two boundary lines of $A$ by $l_{i}=\{i\} \times \mathbb{R}(i=0,1)$. Suppose $l_{0} \subset \partial \widetilde{\mu}$. Hence, $\pi\left(l_{0}\right)$ is a boundary circle of $\mu$, where $\pi: \widetilde{M} \rightarrow M$ is the covering map. This circle $\pi\left(l_{0}\right)$ represents a nontrivial element $g \in \pi_{1}(\mu) \subset \pi_{1}(M)$, and $g$ acts on $\widetilde{M}$ fixing $A \times I$. By our construction, unless $s=1, \beta_{s-1}$ is an arc properly embedded in $A \times\{t\}$ with both endpoints in $l_{1} \times\{t\}$.

If the length of the subarc of $l_{1} \times\{t\}$ between $Z_{s}$ and $Z_{s-1}$ is large, then $g\left(Z_{s}\right)$ (or $g^{-1}\left(Z_{s}\right)$ ) lies between $Z_{s}$ and $Z_{s-1}$ in $l_{1} \times\{t\}$, and hence $\beta_{s-1}$ nontrivially intersects $g\left(\beta_{s-1}\right)$ (or $\left.g^{-1}\left(\beta_{s-1}\right)\right)$. Note that $Z_{s}$ and $g\left(Z_{s}\right)$ lie in puncturing fibers. Since $g \in \pi_{1}(\mu)$ is fixed, the distance between the two puncturing fibers containing $Z_{s}$ and $g\left(Z_{s}\right)$ is relatively small (compared with $k$ ), and hence they puncture the same cross disk. Moreover, since $\beta_{s-1}$ nontrivially intersects $g\left(\beta_{s-1}\right)$ (or $g^{-1}\left(\beta_{s-1}\right)$ ), the two planes containing $Z_{s}$ and $g\left(Z_{s}\right)$ (or $\left.g^{-1}\left(Z_{s}\right)\right)$ intersect each other, which contradicts the 4 -plane property as in Observation 6.4.

Thus, we can assume length of the subarc of $l_{1} \times\{t\}$ between $Z_{s}$ and $Z_{s-1}$ is relatively small. Hence, the distance between $Z_{s}$ and the two puncturing fibers $\partial \alpha \times I$ is relatively small, where $\alpha$ is a 1 -simplex of $\widetilde{\Gamma}$ and $Z_{s-1} \in \alpha \times I$. As in Observation 6.4, the plane in $\widetilde{F}_{k}$ containing $Z_{s} \cup \beta_{s-1}$ and a plane containing a puncturing fiber in $\partial \alpha \times I$ cannot intersect each other. So, as in the case that $A$ is a disk, the arc $\zeta_{s-1}$, which is the arc in $\widetilde{F}_{k} \cap(\alpha \times I)$ containing $Z_{s-1}$, must be a puncturing arc in $\alpha \times I$. Therefore, in any case, $Z_{s-1}$ lies in a puncturing arc.

Then, we apply the argument above to $\beta_{s-2}(s>2)$. Now, $\partial \beta_{s-2}=Z_{s-1} \cup$ $Z_{s-2}$. Note that in this case $Z_{s-1}$ lies in a puncturing arc $\left(Z_{s}\right.$ lies in a puncturing fiber in the case above), but this does not make any difference when using Observation 6.4. Hence, $Z_{s-2}$ lies in a puncturing arc, and inductively, each $Z_{i} \in \beta(1 \leq i \leq s-1)$ lies in a puncturing arc. 
By our assumption before, $Z_{1}$ lies in the sub cross disk $D_{[k / 2]}$. We can choose $k$ large enough so that there is no short arc containing $Z_{1}$ and with endpoints in different components of $\widetilde{\mu} \times \partial I$. This contradicts Claim 2. Thus, Lemma 6.3 holds in the case that $\mu$ is a compact orientable surface.

If $\mu$ is a compact nonorientable surface, since $M$ is orientable, we can apply Hatcher's trick to the horizontal boundary of a twisted $I$-bundle over $\mu$, and the proof is the same.

Suppose $\mu$ contains a noncompact leaf. Let $B$ be a branched surface fully carries $\mu, L$ be the branch locus of $B$, and $p: N(B) \rightarrow B$ be the map that collapses every $I$-fiber of $N(B)$ to a point. We can assume $\partial B$ is a union of circles in $\partial M$. By previous arguments, any such branched surface always fully carries a compact surface with the same boundary slope as $\partial \mu$. Let $S$ be a compact surface fully carried by $B$. By Claim 1 and Hatcher's trick, as in the case that $\mu$ is a compact surface, $F_{k}$ is not transverse to the $I$-fibers of $N(B)$ along any arc of $F_{k} \cap S$. As before, in some tetrahedron $T$, there must be two different types of quadrilateral normal disks in $T \cap S$ and $T \cap F_{k}$ respectively. Thus, after a small homotopy, we can assume that each $I$-fiber of $N(B)$ either transversely intersects $F_{k}$ or entirely lies in $F_{k}$, in which case the local picture of this fiber is as shown in Figure 6.5 and we also call such fibers puncturing fibers. We can assume there are only finitely many puncturing fibers for each $F_{k}$.

$N(B)$ can be viewed as the gluing of a collection of $I$-bundles over compact surfaces along $p^{-1}(L)$. Now, we use the puncturing fibers to decompose $N(B)$ into a similar structure. We say $\alpha \times I \subset N(B)$ is vertical if $\{p\} \times I$ is a subarc of an $I$-fiber of $N(B)$ for each $p \in \alpha$ and $(\alpha \times I) \cap \mu=\alpha \times C$ for some closed set $C \subset I$. We start with the puncturing fibers of $F_{k}$. Since every leaf of $\mu$ is dense, we can add finitely many vertical rectangles $\alpha_{i} \times I \subset N(B)$ $(i=1, \ldots, n)$ such that $\partial \alpha_{i} \times I$ is a pair of subarcs of puncturing fibers for each $i$ and $\mu-\cup_{i=1}^{n} \alpha_{i} \times I$ consists of disks and annular neighborhoods of circles in $\partial \mu$. Moreover, we can assume that there is a union of products $d_{i} \times I(i=1, \ldots, s)$ and $A_{i} \times I(i=1, \ldots, t)$ that are glued along $\cup_{i=1}^{n} \alpha_{i} \times I$, such that:

(1) each $d_{i}$ is a disk and $\partial d_{i} \times I$ lies in $\cup_{i=1}^{n} \alpha_{i} \times I$ for each $i$;

(2) each $A_{i}$ is an annulus, one component of $\partial A_{i} \times I$ lies in $\partial M$ and the other component of $\partial A_{i} \times I$ lies in $\cup_{i=1}^{n} \alpha_{i} \times I$;

(3) $\{p\} \times I$ is a subarc of an $I$-fiber of $N(B)$ for each $p$ in $d_{i}$ or $A_{i}$;

(4) $\mu$ lies in the union of these products $\alpha_{i} \times I$ 's, $d_{i} \times I$ 's and $A_{i} \times I$ 's;

(5) $\mu \cap\left(d_{i} \times I\right)=d_{i} \times C_{i}$ and $\mu \cap\left(A_{i} \times I\right)=A_{i} \times C_{i}^{\prime}$, where $C_{i}$ and $C_{i}^{\prime}$ are closed sets in $\operatorname{int}(I)$. 
Furthermore, we can assume the diameter of $d_{i}$ and the length of $\partial A_{i}$ are bounded by a number independent of the puncturing fibers, since $\mu$ is fixed. In fact, after a small perturbation, we can view the union of these products $\alpha_{i} \times I$ 's, $d_{i} \times I$ 's and $A_{i} \times I$ 's as a fibered neighborhood $N\left(B^{\prime}\right)$ of another branched surface $B^{\prime}$ that also fully carries $\mu$. We can also view $\cup_{i=1}^{n} \alpha_{i} \times I$ as $p^{-1}\left(L^{\prime}\right)$, where $L^{\prime}$ is the branch locus of $B^{\prime}$ and $p: N\left(B^{\prime}\right) \rightarrow B^{\prime}$ is the map collapsing every $I$-fiber to a point.

The new branched surface $B^{\prime}$ also fully carries a compact surface, say $S$. We can suppose $S$ lies in $N\left(B^{\prime}\right)$ and $S$ does not intersect $d_{i} \times \partial I$ or $A_{i} \times \partial I$. By claim 1, we can assume each point in $\partial S \cap \partial F_{k}$ has the same sign. Since the size of each $d_{i}$ is bounded by a number independent of the puncturing fibers, if $k$ is large, there is a cross disk from $F_{k}$ cutting through $d_{i} \times I$ for each $i$. Moreover, we can choose an appropriate surface $S$ so that at least one point of $\partial S \cap \partial F_{k}$ belongs to a sub cross disk $D_{[k / 2]}$ as before. By our construction, $\partial d_{i} \times I$ and $\partial A_{i} \times I$ contain subarcs of puncturing fibers which puncture a cross disk. After some homotopy as in the case that $\mu$ is a compact surface, we can also assume that any $\partial$-parallel arc of $F_{k}$ in $\partial d_{i} \times I$ or $\partial A_{i} \times I$ does not intersect $S$. Then, we can define puncturing arcs using the $d_{i} \times I$ 's and $A_{i} \times I$ 's similar to the case that $\mu$ is a compact surface, and the proof is the same.

Theorem 2, which is a generalization of Hatcher's theorem, now follows easily from Corollary 3.3 and Lemmas 6.1 and 6.3.

Theorem 2 Let $M$ be an orientable and irreducible 3-manifold whose boundary is an incompressible torus, and let $\mathcal{H}$ be the set of injective surfaces that are embedded along their boundaries and satisfy the 4-plane property. Suppose that $M$ does not contain any nonperipheral closed (embedded) incompressible surfaces. Then the surfaces in $\mathcal{H}$ can realize only finitely many slopes.

Proof Suppose that the surfaces can realize infinitely many slopes. Let $\left\{F_{n}\right\}$ be a sequence of surfaces in $\mathcal{H}$ no two of which have the same boundary slopes. Since they have different boundary slopes, by Corollary 3.3, the surfaces in $\left\{F_{n}\right\}$ cannot be fully carried by finitely many immersed branched surfaces. Then, by the argument in section 4 , there exist a sequence of cross disks from $\left\{F_{n}\right\}$ that gives rise to an essential lamination. However, Lemma 6.3 imply that the sequence $\left\{F_{n}\right\}$ contains a subsequence of surfaces with the same boundary slope, which contradicts our assumption that the surfaces in $\left\{F_{n}\right\}$ all have different boundary slopes. 
As an application of Theorem 2, we prove Theorem 3, which gives the first nontrivial examples of 3-manifolds that do not admit any nonpositive cubings. Before we proceed, we prove the following lemma.

Lemma 6.5 Let $M$ be a closed and irreducible 3-manifold, $S$ be a closed least weight surface in $M$ with the 4-plane property, and $C$ be a homotopically nontrivial simple closed curve that intersects $S$ nontrivially. Then $S-C$ is a surface with the 4-plane property in $M-C$.

Proof Let $\widetilde{M}$ be the universal cover of $M$ and $\widetilde{C}$ be the preimage of $C$ in $\widetilde{M}$. So, $\widetilde{M}-\widetilde{C}$ is a cover of $M-C$. Let $\widetilde{S}$ be the preimage of $S$ in $\widetilde{M}$. Then, $\widetilde{S}-\widetilde{C}$ is a collection of embedded surfaces in $\widetilde{M}-\widetilde{C}$. Since $S$ has the $4-$ plane property, among any 4 embedded surfaces in $\widetilde{S}-\widetilde{C}$, there is a disjoint pair. Moreover, as each surface in $\widetilde{S}-\widetilde{C}$ is embedded, among any 4 planes in the preimage of $\widetilde{S}-\widetilde{C}$ in the universal cover of $\widetilde{M}-\widetilde{C}$ (ie the universal cover of $M-C)$, there is a disjoint pair. Therefore, $S-C$ satisfies the 4-plane property in $M-C$.

Theorem 3 Let $M$ be an orientable and irreducible 3-manifold whose boundary is an incompressible torus. Suppose that $M$ does not contain any closed nonperipheral (embedded) incompressible surfaces. Then only finitely many Dehn fillings on $M$ can yield 3-manifolds that admit nonpositive cubings.

Proof Let $M(s)$ be the closed 3-manifold after doing Dehn filling along slope $s$, and $C_{s}$ be the core of the solid torus glued to $M$ during the Dehn filling. Then, except for finitely many slopes, $C_{s}$ is a homotopically nontrivial curve in $M(s)$. Suppose that $M(s)$ admits a nonpositive cubing. For each cube in the cubing, there are 3 disks parallel to the square faces and that intersect the edges of the cube in their mid-points. These mid-disks from all the cubes in the cubing match up and yield a union of immersed surfaces, which we denote by $\mathcal{S}$. The complement of $\mathcal{S}$ is a union of 3-balls. Aitchison and Rubinstein have shown that these surfaces (and their double covers in $M(s)$ if they are one-sided) satisfy the $4-$ plane property [1]. Since $C_{s}$ is nontrivial and the complement of $\mathcal{S}$ is a union of 3-balls, $C_{s}$ must nontrivially intersect at least one immersed surface in $\mathcal{S}$. Let $N\left(C_{s}\right)$ be a small tubular neighborhood of $C_{s}$. Note that $\mathcal{S}-\operatorname{int}\left(N\left(C_{s}\right)\right)$ may not be $\partial$-injective in $M$ and we need to perform some homotopy on the surfaces in $\mathcal{S}$. Similar to the case of embedded incompressible surfaces [29], we push the (immersed) $\partial$-compressing disk across $N\left(C_{s}\right)$ and get less intersection circles. Since $\mathcal{S}$ is immersed, this homotopy changes the intersection patterns of $\mathcal{S}$ in $M(s)$, but by choosing innermost $\partial$-compressing 
disks if necessary, we can require that the disjoint planes in the preimage of $\mathcal{S}$ in the universal cover of $M(s)$ remain disjoint after this homotopy, and hence this homotopy preserves the 4 -plane property of $\mathcal{S}$.

The nonpositive cubing gives $M(s)$ a singular nonpositive metric and $\mathcal{S}$ consists of totally geodesic surfaces in this singular metric [1]. The geodesic that represents $C_{s}$ must intersect some (totally geodesic) surface in $\mathcal{S}$. Since the singular metric is nonpositive, after the homotopy above, $C_{s}$ must still intersect some immersed surface in $\mathcal{S}$. Hence, by Lemma 6.5 , there is an injective surface in $M$ that satisfies the 4 -plane property and has boundary slope $s$. By Theorem 2, there are only finitely many such slopes. Therefore, the theorem holds.

\section{References}

[1] I R Aitchison, J H Rubinstein, An introduction to polyhedral metrics of nonpositive curvature on 3-manifolds, from: "Geometry of low-dimensional manifolds, 2 (Durham, 1989)", London Math. Soc. Lecture Note Ser. 151, Cambridge Univ. Press, Cambridge (1990) 127-161

[2] M Baker, On boundary slopes of immersed incompressible surfaces, Ann. Inst. Fourier (Grenoble) 46 (1996) 1443-1449

[3] M Baker, D Cooper, Immersed, Virtually-Embedded, Boundary Slopes, Topology Appl. 102 (2000) 239-252

[4] A Candel, Laminations with transverse structure, Topology 38 (1999) 141-165

[5] Y Choi, (3,1) surfaces via branched surfaces, Thesis, Caltech (1998)

[6] J Christy, Immersing branched surfaces in dimension three, Proc. Amer. Math. Soc. 115 (1992) 853-861

[7] M Culler, W Jaco, H Rubinstein, Incompressible surfaces in once-punctured torus bundles, Proc. London Math. Soc. 45 (1982) 385-419

[8] W Floyd, A Hatcher, Incompressible surfaces in punctured-torus bundles, Topology Appl. 13 (1982) 263-282

[9] W Floyd, U Oertel, Incompressible surfaces via branched surfaces, Topology 23 (1984) 117-125

[10] M Freedman, J Hass, P Scott, Least area incompressible surfaces in 3manifolds, Invent. Math. 71 (1983) 609-642

[11] D Gabai, U Oertel, Essential laminations in 3-manifolds, Ann. of Math. (2) 130 (1989) 41-73

[12] M Gromov, Hyperbolic groups, Essays in group theory, MSRI Pubs. 8, 75-264

[13] W Haken, Theorie der Normal Flachen, Acta. Math. 105 (1961) 245-357 
[14] J Hass, P Scott, Homotopy equivalence and homeomorphism of 3-manifolds, Topology 31 (1992) 493-517

[15] A Hatcher, On the boundary curves of incompressible surfaces, Pacific J. Math. 99 (1982) 373-377

[16] A Hatcher, W Thurston, Incompressible surfaces in 2-bridge knot complements, Invent. Math. 79 (1985) 225-246

[17] H Imanishi, On the theorem of Denjoy-Sacksteder for codimension one foliations without holonomy, J. Math. Kyoto Univ. 14 (1974) 607-634

[18] W Jaco, H Rubinstein, PL minimal surfaces in 3-manifolds, J. Differential Geom. 27 (1988) 493-524

[19] W Jaco, H Rubinstein, 0-efficient triangulations of 3-manifolds, preprint

[20] H Kneser, Geschlossene Flachen in Dreidimensionalen Mannigfaltigkeiten, Jahres. der Deut. Math. Verein. 38 (1929) 248-260

[21] T Li, An algorithm to find vertical tori in small Seifert fiber spaces, e-print: arXiv:math.GT/0209107

[22] J Maher, Virtually embedded boundary slopes, Topology Appl. 95 (1999) 63-74

[23] L Mosher, Geometry of cubulated 3-manifolds, Topology 34 (1995) 789-814

[24] L Mosher, U Oertel, Spaces which are not negatively curved, Comm. Anal. Geom. 6 (1998) 67-140

[25] U Oertel, Incompressible branched surfaces, Invent. Math. 76 (1984) 385-410

[26] U Oertel, Boundaries of injective surfaces, Topology Appl. 78 (1997) 215-234

[27] U Oertel, Measured laminations in 3-manifolds, Trans. Amer. Math. Soc. 305 (1988) 531-573

[28] H Rubinstein, M Sageev, Intersection patterns of essential surfaces in 3manifolds, Topology 38 (1999) 1281-1291

[29] W Thurston, The geometry and topology of three-manifolds, Lecture notes. Princeton University $(1977 / 78)$

[30] F Waldhausen, On irreducible 3-manifolds which are sufficiently large, Ann. of Math. 871968 56-88 